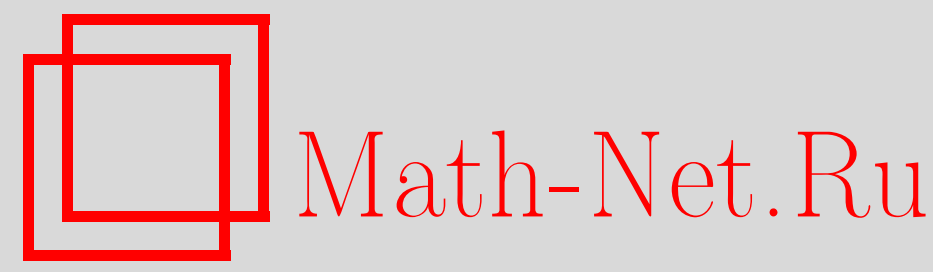

Р. Р. Гадыльшин, О задаче Дирихле для уравнения Гельмгольца на плоскости с граничными условиями на почти замкнутой кривой, Матем. сб., 2000, том 191, номер 6, 43-68

DOI: https://doi.org/10.4213/sm483

Использование Общероссийского математического портала Math-Net.Ru подразумевает, что вы прочитали и согласны с пользовательским соглашением

http://www.mathnet.ru/rus/agreement

Параметры загрузки:

IP : 34.227 .88 .159

26 апреля 2023 г., 13:05:26 


\title{
Р.Р. Гадыльшин \\ О задаче Дирихле для уравнения Гельмгольца на плоскости с граничными условиями на почти замкнутой кривой
}

\begin{abstract}
В работе рассмотрена двумерная краевая задача Дирихле для оператора Гельмгольца с граничньми условиями на почти замкнутой кривой $\Gamma_{\varepsilon}$, где $\varepsilon \ll 1$ - расстояние между концами кривой. Построена полная асимптотика полюса аналитического продолжения функции Грина этой задачи, сходящегося к простой собственной частоте предельной внутренней задачи, в случае, когда соответствующая собственная функция предельной задачи имеет нуль второго порядка в центре сжатия отверстия. Исследовано влияние симметрии щели на величину мнимой части полюсов.
\end{abstract}

Библиографиия: 30 названий.

\section{§ 1. Постановка задачи и предварительные сведения}

Пусть $\Omega$ - ограниченная односвязная область на плоскости $x=\left(x_{1}, x_{2}\right)$, ее граница $\Gamma_{0} \in C^{\infty}, \omega_{\varepsilon}$ - открытая связная часть $\Gamma_{0}$, имеющая длину порядка $\varepsilon$, $0<\varepsilon \ll 1-$ мальй параметр, $\Gamma_{\varepsilon}=\Gamma_{0} \backslash \overline{\omega_{\varepsilon}}$. В работе рассматривается следующая краевая задача:

$$
\begin{gathered}
\left(\Delta+k^{2}\right) E_{\varepsilon}=f, \quad x \notin \overline{\Gamma_{\varepsilon}}, \quad E_{\varepsilon}=0, \quad x \in \Gamma_{\varepsilon}, \\
E_{\varepsilon}=O\left(r^{-1 / 2}\right), \quad \frac{\partial E_{\varepsilon}}{\partial r}-i k E_{\varepsilon}=o\left(r^{-1 / 2}\right), \quad r \rightarrow \infty,
\end{gathered}
$$

где $r=|x|, k$ - положительное число, $f$ - функция из $L_{2}\left(\mathbb{R}^{2}\right)$ с ограниченным носителем. Из [1]-[3] следует, что краевая задача (1.1), (1.2) однозначно разрешима в классе функций, принадлежащих $W_{2, \text { loc }}^{1}\left(\mathbb{R}^{2}\right)$, что соответствует выполнению условий Мейкснера [4] в окрестности концов кривой $\Gamma_{\varepsilon}$. Под пространствами $X_{\text {loc }}(D)$ понимается следуюшее. Пусть $S(R)$ - круг радиуса $R$ с центром в начале координат, а $X$ - символ какого-либо банахова пространства. Тогда

$$
X_{\text {loc }}(D) \stackrel{\text { def }}{=}\{u: u \in X(D \cap S(R)) \forall R\} .
$$

Будем также говорить, что последовательность сходится в $X_{10 c}(D)$, если она сходится в $X(D \cap S(R))$ для любого $R$.

Краевая задача (1.1), (1.2) является электромагнитньм аналогом акустического резонатора Гельмгольца [5]-[7]. Ее физический смысл можно интерпретировать (см., например, [3], [8], [9]) как излучение (supp $f \subset \Omega$ ) или рассеяние

Работа выполнена при финансовой поддержке Российского фонда фундаментальных исследований (грант № 96-01-00501).

(C) Р. Р. ГАдыльшин 2000 
$(\operatorname{supp} f \cap \bar{\Omega}=\varnothing)$-поляризованных полей для цилиндрических идеально проводящих поверхностей, сечением которых является $\Gamma_{\varepsilon}$, а малость $\omega_{\varepsilon}$ соответствует узкой шели. Очевидно, что задача рассеяния плоской $E$-поляризованной волны сводится к задаче (1.1), (1.2) с финитной правой частью $f$, носитель которой лежит вне $\bar{\Omega}$.

Для фиксированного $k$ такого, что $k^{2}$ не является собственным значением предельной внутренней задачи

$$
\left(\Delta+k^{2}\right) E_{0}^{\text {in }}=f^{\text {in }}, \quad x \in \Omega, \quad E_{0}^{\text {in }}=0, \quad x \in \Gamma_{0},
$$

решение $(1.1),(1.2)$ при $\varepsilon \rightarrow 0$ сходится к $E_{0}^{\text {in }}$ в $\Omega\left(\right.$ если $f^{\text {in }}-$ сужение $f$ на $\left.\Omega\right)$ и к решению предельной внешней задачи

$$
\begin{gathered}
\left(\Delta+k^{2}\right) E_{0}^{\mathrm{ex}}=f^{\mathrm{ex}}, \quad x \in \Omega^{\mathrm{ex}}, \quad E_{0}^{\mathrm{ex}}=0, \quad x \in \Gamma_{0}, \\
E_{0}^{\mathrm{ex}}=O\left(r^{-1 / 2}\right), \quad \frac{\partial E_{0}^{\mathrm{ex}}}{\partial r}-i k E_{0}^{\mathrm{ex}}=o\left(r^{-1 / 2}\right), \quad r \rightarrow \infty,
\end{gathered}
$$

где $f^{\text {ех }}$ - сужение $f$ на $\Omega^{\mathrm{ex}}=\mathbb{R}^{2} \backslash \bar{\Omega}$ (см. [10]-[12]).

Известно также [11], [12], что функция Грина $G_{\varepsilon}(x, y, k)$ задачи $(1.1),(1.2)$ (а следовательно, и ее решение) допускает аналитическое продолжение в комплексную плоскость с разрезом вдоль отрицательной полуоси, которое имеет полюсы только в нижней полуплоскости и которое в любой окрестности собственной частоты $k_{0}$ при любом достаточно малом $\varepsilon$ имеет по крайней мере один полюс $\tau_{\varepsilon}$. С одной стороны, “сползание” полюсов возмущенной задачи в нижнюю полуплоскость обеспечивает разрешимость (1.1), (1.2) при вещественных $k$. С другой стороны, близость части из них к собственным частотам предельной внутренней задачи существенно сказывается на решении (1.1), (1.2) при $k \subset \mathbb{R}$, близких к $k_{0}$. В частности, в [12] показано, что если $k_{0}^{2}$ является простым собственным значением предельной внутренней задачи, а $\psi$ - соответствуюшая нормированная в $L_{2}(\Omega)$ собственная функция, продолженная нулем в $\Omega^{\mathrm{ex}}$, то при комплексных $k$, близких к $k_{0}$, для аналитического продолжения решения краевой задачи (1.1), (1.2) справедливо представление

$$
E_{\varepsilon}(x, k)=-\frac{\Psi_{\varepsilon}(x)}{\tau_{\varepsilon}^{2}-k^{2}} \int_{\mathbb{R}^{2}} \Psi_{\varepsilon}(y) f(y) d y+\widetilde{E}_{\varepsilon}(x, k),
$$

где полюс $\tau_{\varepsilon} \rightarrow k_{0}$ при $\varepsilon \rightarrow 0$, функция $\Psi_{\varepsilon}$ сходится к $\psi$ при $\varepsilon \rightarrow 0$ в $W_{2, \text { lос }}^{1}\left(\mathbb{R}^{2}\right)$, является решением уравнения $\left(\Delta+\tau_{\varepsilon}^{2}\right) \Psi_{\varepsilon}=0$ вне $\overline{\Gamma_{\varepsilon}}$, удовлетворяющим однородному граничному условию Дирихле на $\Gamma_{\varepsilon}$, а $\widetilde{E}_{\varepsilon}-$ голоморфная в некоторой окрестности точки $k_{0}$ функция из $W_{2, \text { loc }}^{1}\left(\mathbb{R}^{2}\right)$, которая сходится к аналитическому продолжению функции $E_{0}^{\mathrm{ex}}$ равномерно по $k$ в $W_{2,1 \text { сос }}^{1}\left(\Omega^{\mathrm{ex}}\right)$ и для которой справедлива равномерная по $\varepsilon$ и $k$ оценка

$$
\left\|\widetilde{E}_{\varepsilon}\right\|_{1, S(R)} \leqslant C_{R}\|f\|_{\mathbb{R}^{2}}
$$

где $\|\cdot\|_{1, Q}$ и $\|\cdot\|_{Q}-$ нормы в $W_{2}^{1}(Q)$ и $L_{2}(Q)$ соответственно, а $R$ - любое число такое, что supp $f \subset S(R)$.

Под голоморфной функцией из $X_{\text {loc }}(D)$ понимается функция, голоморфная как элемент из $X(D \cap S(R))$ для любого $R$. По аналогии с внутренней предельной 
задачей будем называть функцию $\Psi_{\varepsilon}$ собственной функцией (1.1), (1.2). Ясно, что при любом фиксированном $\varepsilon$ эта собственная функция экспоненциально растет на бесконечности. (Можно показать, что она удовлетворяет обобшенному условию излучения [13] на бесконечности.)

Из (1.3) и (1.4) видно, что первое слагаемое в правой части (1.3) (которое будем называть резонанснылм членом) при вещественных $k$ принимает наибольшие значения в случае, когда

$$
k=k(\varepsilon)=\operatorname{Re} \tau_{\varepsilon}+O\left(\operatorname{Im} \tau_{\varepsilon}\right)
$$

Решения (1.1), (1.2) при $k$, удовлетворяющих (1.5), будем называть решениями в пиковом режиме. Однако, так как $\Psi_{\varepsilon} \rightarrow 0$ в $\Omega^{\text {ex }}$ при $\varepsilon \rightarrow 0$, то непосредственно из (1.3) не видно, насколько сушественное влияние оказывает резонансный член даже в пиковом режиме на решение задачи рассеяния (или задачи излучения в $\Omega^{\mathrm{ex}}$ ). Поэтому для выяснения этого вопроса необходим дополнительный асимптотический анализ $\tau_{\varepsilon}$ и $\Psi_{\varepsilon}$.

В случае, когда

A. $\Omega$ совпадает в окрестности начала координат с полуплоскостью $x_{2}>0$, а $\omega_{\varepsilon}-$ интервал $\left(\varepsilon \omega_{-}, \varepsilon \omega_{+}\right)$на прямой $x_{2}=0$, где $\omega_{-}<\omega_{+}-$произвольнье действительные числа,

такой анализ проведен в [11] при дополнительном предположении $|\nabla \psi(0)| \neq 0$. Было показано, что в этом случае

$$
\operatorname{Im} \tau_{\varepsilon}=-\varepsilon^{4}\left(a_{1}^{2}+O(\varepsilon)\right), \quad \Psi_{\varepsilon}(x) \sim \varepsilon^{2} a_{2} G_{1}^{\mathrm{ex}}\left(x, 0, k_{0}\right), \quad x \in \Omega^{\mathrm{ex}} \backslash S\left(\varepsilon^{1 / 2}\right),
$$

где $a_{j} \neq 0$ - некоторые явно вычисляемые постоянные,

$$
G_{1}^{\mathrm{ex}}(x, 0, k)=\left.\frac{\partial}{\partial y_{2}} G^{\mathrm{ex}}(x, y, k)\right|_{y=0},
$$

$\mathrm{a} G^{\mathrm{ex}}(x, y, k)$ - функция Грина предельной внешней задачи. Асимптотики (1.6) делают представление (1.3) конструктивным. Из (1.3), (1.6) следует, что в пиковом режиме решение задачи рассеяния имеет порядок $\varepsilon^{-2}$ в $\Omega$, а в $\Omega^{\text {ех } о т л и ч а е т с я ~ о т ~}$ решения предельной внешней задачи на величину $O(1)$. Для задачи же излучения решение в пиковом режиме будет иметь уже порядки $\varepsilon^{-4}$ и $\varepsilon^{-2}$ в $\Omega$ и $\Omega^{\text {ex }}$ соответственно.

Однако, если $|\nabla \psi(0)|=0$, то $a_{j}=0$ (см. [11]) и асимптотики (1.6) теряют свою эффективность.

Построению полных асимптотических разложений полюсов $\tau_{\varepsilon}$ и соответствующих им собственных функций $\Psi_{\varepsilon}$, позволяющих эффективно использовать представление (1.3) и в таком вырожденном случае, посвящена настоящая работа. Заметим, что выполнение или невыполнение условия $|\nabla \psi(0)|=0$ можно интерпретировать как выбор места прорезания шели.

Работа имеет следующую структуру. В $\S 2$ приведены формулировки основных утверждений и, в частности, дано их приложение к задаче рассеяния плоской волны на резонаторе. В $\S 3$ исследован случай, когда отверстие $\omega_{\varepsilon}$ несимметрично относительно начала координат, а в $\S 4$ построены асимптотики для симметричного $\omega_{\varepsilon}$. Показано, что рассмотренные в этих параграфах ситуации достаточно 
сильно отличаются как друг от друга, так и от невырожденного случая, рассмотренного в [11], что выражается, в первую очередь, в разных порядках малости мнимых частей полюсов, соответствующих потерям энергии в физике. В заключительном $\S 5$ обсуждаются вопросы, имеющие косвенное отношение к основным результатам работы.

\section{§ 2. Формулировка результатов}

Всюду в дальнейшем предполагается, что $\Omega$ удовлетворяет условию А из предыдушего параграфа, $k_{0}^{2}$ - простое собственное значение, а соответствующая собственная функция $\psi$ имеет в начале координат нуль второго порядка. В силу этих предположений, уравнения Гельмгольца и граничных условий Дирихле асимптотика в нуле собственной функции имеет вид

$\psi(x)=\psi_{2} x_{1} x_{2}+\frac{1}{6} \psi_{3}\left(3 x_{2} x_{1}^{2}-x_{2}^{3}\right)+\psi_{4} x_{1} x_{2}\left(x_{1}^{2}-x_{2}^{2}\right)-\frac{1}{12} k_{0}^{2} \psi_{2} x_{1} x_{2} r^{2}+O\left(r^{5}\right)$, где $\psi_{j}$ - некоторые постоянные, зависящие от геометрии области $\Omega$, и $\psi_{2} \neq 0$.

Асимптотики полюса $\tau_{\varepsilon}$ и соответствуюшей собственной функции $\Psi_{\varepsilon}$ строятся методом согласования асимптотических разложений [14]. Главные члены асимптотик $\tau_{\varepsilon}$ и $\Psi_{\varepsilon}$ зависят от некоторых вполне определенных функций и постоянных. Обозначим

$$
\begin{aligned}
G_{2}^{\mathrm{ex}}(x, 0, k) & =\left.\frac{\partial^{2}}{\partial y_{1} \partial y_{2}} G^{\mathrm{ex}}(x, y, k)\right|_{y=0}, \\
\sigma_{i, i}(k) & =\lim _{R \rightarrow \infty} \int_{\partial S(R)}\left|G_{i}^{\mathrm{ex}}(x, 0, k)\right|^{2} d s \\
\sigma_{1,2}(k) & =\sigma_{2,1}(k)=\lim _{R \rightarrow \infty} \operatorname{Re} \int_{\partial S(R)} G_{1}^{\mathrm{ex}}(x, 0, k) \overline{G_{2}^{\mathrm{ex}}(x, 0, k)} d s, \quad k>0 .
\end{aligned}
$$

Положительные постоянные $\sigma_{i i}(k)$ обычно называют поперечниками рассеяния полей $G_{i}^{\mathrm{ex}}(x, 0, k)(\mathrm{cm} .[6],[15])$.

Ниже (в $§ 4)$ будет доказана

ЛЕмма 2.1. Квадратичная форма

$$
\left\langle z, \sigma_{\Omega} z\right\rangle=\sum_{j, i=1}^{2} \sigma_{i j} z_{i} z_{j}
$$

где $\sigma_{i, j}=\sigma_{i, j}\left(k_{0}\right)$, является полохсительно определенной.

В окрестности отверстия $\omega_{\varepsilon}$ главные члены асимптотики собственных функций выражаются через функцию

$$
\begin{array}{r}
Y_{2}(\xi)=\frac{1}{2} \operatorname{Im}\left(\zeta^{2}+\zeta\left(\left(\zeta-\omega_{-}\right)\left(\zeta-\omega_{+}\right)\right)^{1 / 2}\right)+2 \varkappa_{\omega} Y_{1}(\xi), \\
\text { где } \xi=\left(\xi_{1}, \xi_{2}\right), \varkappa_{\omega}=\frac{\omega_{+}+\omega_{-}}{2}, \zeta=\xi_{1}+i \xi_{2}(i-\text { мнимая единица }), \\
Y_{1}(\xi)=\frac{1}{2} \operatorname{Im}\left(\zeta+\left(\left(\zeta-\omega_{-}\right)\left(\zeta-\omega_{+}\right)\right)^{1 / 2}\right),
\end{array}
$$

а разрез комплексной плоскости взят по $\gamma=\left\{\xi=\left(\xi_{1}, 0\right):\left(\xi_{1}<\omega_{-}\right) \cup\left(\xi_{1}>\omega_{+}\right)\right\}$.

Обозначим через $[N]$ целую часть $N$ и положим $c_{\omega}=\left(\frac{\omega_{+}-\omega_{-}}{4}\right)^{2}$. Основным содержанием настоящей работы являются следующие два утверждения, доказательство которых будет приведено в $\S 3$ и $\S 4$ соответственно. 
ТЕОРема 2.1. Если $\omega_{-} \neq-\omega_{+}$, то асимптотики полюса, сходящегося $\kappa k_{0}$, и соответствующей собственной функции имеют вид

$$
\begin{gathered}
\tau_{\varepsilon}=k_{0}+\sum_{j=4}^{\infty} \sum_{i=0}^{[j / 2]-2} \tau_{j, i} \varepsilon^{j} \ln ^{i} \varepsilon \\
\tau_{4,0}=-\frac{1}{4 k_{0}} \pi \psi_{2}^{2} c_{\omega}\left(c_{\omega}+2 \varkappa_{\omega}^{2}\right), \\
\tau_{6,1}=\frac{1}{2} \pi k_{0}\left(\psi_{2} c_{\omega} \varkappa_{\omega}\right)^{2}, \quad \tau_{5,0}=-\frac{1}{k_{0}} \pi \psi_{2} \psi_{3} \varkappa_{\omega} c_{\omega}\left(2 c_{\omega}+\varkappa_{\omega}^{2}\right), \\
\Psi_{\varepsilon}(x) \sim \tau_{6,0}=-\frac{1}{2}\left(\pi c_{\omega} \varkappa_{\omega} \psi_{2}\right)^{2} \sigma_{1,1}, \\
\Psi_{\varepsilon}(x) \sim \frac{1}{2} \varepsilon^{2} \psi_{2} Y_{2}\left(\frac{x}{\varepsilon}\right) \quad n p u \quad x \in \Omega \backslash S\left(\varepsilon^{1 / 2}\right), \\
\Psi_{\varepsilon}(x) \sim-\varepsilon^{3} \pi \psi_{2} c_{\omega} \varkappa_{\omega} G_{1}^{\mathrm{ex}}\left(x, 0, k_{0}\right) \quad n p u \quad x \in S\left(2 \varepsilon^{1 / 2}\right), \\
n p u \quad x \in \Omega^{\operatorname{ex}} \backslash S\left(\varepsilon^{1 / 2}\right)
\end{gathered}
$$

в $W_{2, \mathrm{loc}}^{1}\left(\mathbb{R}^{2}\right)$ (m.е. $\quad$ в $W_{2}^{1}\left(\Omega \backslash \overline{S\left(\varepsilon^{1 / 2}\right)}\right), W_{2}^{1}\left(S\left(2 \varepsilon^{1 / 2}\right)\right)$ и $W_{2, \mathrm{loc}}^{1}\left(\Omega^{\mathrm{ex}} \backslash \overline{S\left(\varepsilon^{1 / 2}\right)}\right)$ соответственно).

ТЕОРема 2.2. Если $\omega_{-}=-\omega_{+}$, то асимптотики полюса, сходящегося $\kappa k_{0}$, и соответствующей собственной функции имеют вид

$$
\begin{aligned}
& \tau_{\varepsilon}=k_{0}+\sum_{j=2}^{\infty} \sum_{i=0}^{[j / 2]-1} \tau_{2 j, i} \varepsilon^{2 j} \ln ^{i} \varepsilon \\
& \tau_{4,0}=-\frac{1}{4 k_{0}} \pi\left(\psi_{2} c_{\omega}\right)^{2}, \quad \tau_{6,0}=-\frac{\pi c_{\omega}^{3}}{k_{0}}\left(\psi_{4} \psi_{2}+\frac{1}{6} \psi_{3}^{2}-\frac{1}{24} k_{0}^{2} \psi_{2}^{2}\right), \\
& \tau_{8,1}=\frac{1}{32} \pi k_{0}^{3} c_{\omega}^{4} \psi_{2}^{2}, \\
& \operatorname{Im} \tau_{8,0}=-\frac{1}{8}\left(\pi c_{\omega}^{2}\right)^{2}\left\langle z, \sigma_{\Omega} z\right\rangle, \quad z=\left(\psi_{3}, \psi_{2}\right),
\end{aligned}
$$

Из теорем 2.1, 2.2 вытекает, что пиковые режимы описываются вещественными $k$, представимыми в виде

$$
\begin{array}{lll}
k=k_{0}+\varepsilon^{4} \tau_{4,0}+\varepsilon^{5} \tau_{5,0}+\varepsilon^{6}\left(\tau_{6,1} \ln \varepsilon+t+o(1)\right) & \text { при } & \omega_{-} \neq-\omega_{+}, \\
k=k_{0}+\varepsilon^{4} \tau_{4,0}+\varepsilon^{6} \tau_{6,0}+\varepsilon^{8}\left(\tau_{8,1} \ln \varepsilon+t+o(1)\right) & \text { при } & \omega_{-}=-\omega_{+}
\end{array}
$$


соответственно, где $t$ - любые действительные числа. Подставляя асимптотики $\tau_{\varepsilon}$ и $\Psi_{\varepsilon}$ в $(1.3)$, легко видеть, что в пиковом режиме решения неограниченно растут как в $\Omega$, так и в $\Omega^{\mathrm{ex}}$, но порядки роста различны для случаев $\omega_{-} \neq-\omega_{+}$ и $\omega_{-}=-\omega_{+}$. В пиковых режимах решения задач рассеяния имеют следующие асимптотики:

$$
\begin{array}{rlrl}
E_{\varepsilon}(x, k) \sim \varepsilon^{-3} T_{1}\left(E_{0}^{\mathrm{ex}}, t\right) \psi(x) & \text { при } x \in \Omega \backslash S\left(\varepsilon^{1 / 2}\right), \\
E_{\varepsilon}(x, k) \sim \varepsilon^{-1} \frac{1}{2} \psi_{2} T_{1}\left(E_{0}^{\mathrm{ex}}, t\right) Y_{2}\left(\frac{x}{\varepsilon}\right) & \text { при } x \in S\left(2 \varepsilon^{1 / 2}\right), \\
E_{\varepsilon}(x, k) \sim-\pi \psi_{2} c_{\omega} \varkappa_{\omega} T_{1}\left(E_{0}^{\mathrm{ex}}, t\right) G_{1}^{\mathrm{ex}}\left(x, 0, k_{0}\right) & & \\
& +E_{0}^{\mathrm{ex}}\left(x, k_{0}\right) & \text { при } x \in \Omega^{\mathrm{ex}} \backslash S\left(\varepsilon^{1 / 2}\right),
\end{array}
$$

где

$$
T_{1}\left(E_{0}^{\mathrm{ex}}, t\right)=\left.\frac{\pi \psi_{2} c_{\omega} \varkappa_{\omega}}{2 k_{0}\left(\tau_{6,0}-t\right)} \frac{\partial}{\partial x_{2}} E_{0}^{\mathrm{ex}}\left(x, k_{0}\right)\right|_{x=0}
$$

для несимметричного отверстия $\left(\omega_{-} \neq-\omega_{+}\right)$и

$$
\begin{array}{rlrl}
E_{\varepsilon}(x, k) \sim \varepsilon^{-4} T_{2}\left(E_{0}^{\mathrm{ex}}, t\right) \psi(x) & \text { при } & x \in \Omega \backslash S\left(\varepsilon^{1 / 2}\right), \\
E_{\varepsilon}(x, k) \sim \varepsilon^{-2} \frac{1}{2} \psi_{2} T_{2}\left(E_{0}^{\mathrm{ex}}, t\right) Y_{2}\left(\frac{x}{\varepsilon}\right) & \text { при } \quad x \in S\left(2 \varepsilon^{1 / 2}\right), \\
E_{\varepsilon}(x, k) \sim & -\frac{1}{2} \pi c_{\omega}^{2} T_{2}\left(E_{0}^{\mathrm{ex}}, t\right)\left(\psi_{2} G_{2}^{\mathrm{ex}}\left(x, 0, k_{0}\right)\right. & & \\
& \left.+\psi_{3} G_{1}^{\mathrm{ex}}\left(x, 0, k_{0}\right)\right)+E_{0}^{\mathrm{ex}}\left(x, k_{0}\right) & \text { при } \quad x \in \Omega^{\mathrm{ex}} \backslash S\left(\varepsilon^{1 / 2}\right),
\end{array}
$$

где

$$
T_{2}\left(E_{0}^{\mathrm{ex}}, t\right)=\frac{\pi c_{\omega}^{2}}{4 k_{0}\left(\tau_{8,0}-t\right)}\left(\left.\psi_{2} \frac{\partial^{2}}{\partial x_{1} \partial x_{2}} E_{0}^{\mathrm{ex}}\left(x, k_{0}\right)\right|_{x=0}+\left.\psi_{3} \frac{\partial}{\partial x_{2}} E_{0}^{\mathrm{ex}}\left(x, k_{0}\right)\right|_{x=0}\right),
$$

для симметричного отверстия $\left(\omega_{-}=-\omega_{+}\right)$.

Из (2.5), (2.6), (2.8) и (2.9) видно, что решение задачи рассеяния внутри резонатора и в окрестности отверстия имеет разные порядки роста для случаев несимметричной и симметричной щелей. В свою очередь, из (2.7) и (2.10) следует, что вне резонатора решение отличается от решения предельной внешней задачи на $O(1)$ в обоих случаях, но вид этих добавок различен.

В заключение параграфа покажем, что формулы (2.5)-(2.10) остаются справедливыми и для задачи рассеяния плоской волны $E$ на $\Gamma_{\varepsilon}$. Как известно, решения $E_{\varepsilon}$ и $E_{0}$ задач рассеяния плоской волны на $\Gamma_{\varepsilon}$ и на $\Omega$ - это функции

$$
E_{\delta}(x, k)=E(x, k)+E_{\delta}^{\mathrm{ext}}(x, k), \quad \delta \geqslant 0,
$$

где $E_{\varepsilon}^{\mathrm{ext}}$ и $E_{0}^{\mathrm{ext}}$ - решения уравнения Гельмгольца вне $\overline{\Gamma_{\varepsilon}}$ и в $\Omega^{\mathrm{ex}}$, удовлетворяющие условию излучения (1.2) и граничному условию $E_{\varepsilon}^{\mathrm{ext}}=-E$ и $E_{0}^{\mathrm{ext}}=-E$ на $\Gamma_{\varepsilon}$ и $\partial \Omega$ соответственно. Пусть $\chi(t)$ - бесконечно дифференцируемая срезающая функция, тождественно равная единице при $t<1$ и нулю при $t>2$, а $L>0$ - достаточно большое число такое, что $\bar{\Omega} \subset S(L)$. Очевидно, что функцию $E_{\delta}$ можно представить и в виде

$$
E_{\delta}(x, k)=E(x, k)\left(1-\chi\left(r L^{-1}\right)\right)+E_{\delta, \chi}(x, k),
$$


где $E_{\varepsilon, \chi}-$ решение $(1.1),(1.2)$, а $E_{0, \chi}-$ решение предельной задачи в $\Omega^{\text {ex }}$ с правыми частями, равными

$$
E \Delta_{\chi}+2 \sum_{i=1}^{3} \frac{\partial \chi}{\partial x_{i}} \frac{\partial E}{\partial x_{i}}
$$

Так как носитель правой части лежит в $\Omega^{\mathrm{ex}}$, то для $E_{\varepsilon, \chi}$ справедливы все выше приведенные формулы $(2.5)-(2.10)$ (с заменой в их правых частях $E_{0}^{\text {ex }}$ на $\left.E_{0, \chi}\right)$. Далее, так как $\{0\} \in \partial \Omega$ и по определению $E_{0}=E_{0, \chi}$ в окрестности $\partial \Omega$, то $T_{i}\left(E_{0, \chi}, t\right)=T_{i}\left(E_{0}, t\right)$. Прибавляя к обеим частям представления $(1.3)$ для $E_{\varepsilon, \chi}$ функцию $E(x, k)\left(1-\chi\left(r L^{-1}\right)\right)$, в силу (2.11) получаем, что для решения задачи рассеяния плоской волны остаются справедливыми соотношения $(2.5)-(2.10)$ с заменой в их правых частях функции $E_{0}^{\mathrm{ex}}$ на решение $E_{0}$ задачи рассеяния плоской волны на $\Omega$.

\section{§ 3. Построение асимптотик в случае несимметричного отверстия}

В этом параграфе строятся асимптотики полюса $\tau_{\varepsilon}$ и соответствующей ему собственной функции в случае, когда $\omega_{-} \neq-\omega_{+}$. Обозначим

$$
D_{y}^{j}=\frac{\partial^{j}}{\partial y_{1}^{j-1} \partial y_{2}}
$$

Лемма 3.1. При $k=\mathbb{C}$, близких $\kappa k_{0}$, для функиий Грина $G^{\text {in }}(x, y, k)$ $u G^{\mathrm{ex}}(x, y, k)$ предельных внутренней и внешней задач и их производных справедливы представления

$$
\begin{aligned}
D_{y}^{j} G^{\mathrm{in}}(x, 0, k) & =\frac{\psi(x)}{k_{0}^{2}-k^{2}} D_{x}^{j} \psi(0)+(-1)^{j} \frac{i}{2} D_{x}^{j} \mathscr{H}_{0}^{(1)}(k r)+g_{j}^{\mathrm{in}}(x, k), \\
D_{y}^{j} G^{\mathrm{ex}}(x, 0, k) & =(-1)^{j} \frac{i}{2} D_{x}^{j} \mathscr{H}_{0}^{(1)}(k r)+g_{j}^{\mathrm{ex}}(x, k),
\end{aligned}
$$

где $\mathscr{H}_{0}^{(1)}$ - функиия Ханкеля первого рода, а $g_{j}^{\mathrm{in}}$ и $g_{j}^{\mathrm{ex}}-$ голоморфные функции соответственно из $C^{m}(\bar{\Omega})$ и $C_{\mathrm{loc}}^{m}\left(\overline{\Omega^{\mathrm{ex}}}\right)$ для любых $m$, равные нулю на $\Gamma_{0} \cap S(R)$ для достаточно малого $R$.

ДокАЗАТЕЛЬство. При малых $у$ будем искать функции Грина в виде

$$
G^{\mathrm{in}, \mathrm{ex}}(x, y, k)=\frac{i}{4}\left(\mathscr{H}_{0}^{(1)}(k|x-y|)+\mathscr{H}_{0}^{(1)}\left(k\left|x-y_{*}\right|\right)\right)+\widetilde{G}^{\mathrm{in}, \mathrm{ex}}(x, y, k),
$$

где $y_{*}=\left(y_{1},-y_{2}\right)$.

Покажем сначала справедливость леммы для предельной внутренней задачи. Из представления (3.1) следует, что функция $\widetilde{G}_{j}^{\text {in }}(x, k)=D_{y}^{j} \widetilde{G}^{\text {in }}(x, 0, k)$ принадлежит $C^{m}(\bar{\Omega})$ для $k \neq k_{0}$ и любого $m$, а краевая задача для этой функции имеет вид

$$
\left(\Delta+k^{2}\right) \widetilde{G}_{j}^{\text {in }}=0 \text { при } x \in \Omega, \quad \widetilde{G}_{j}^{\text {in }}=f_{j} \text { при } x \in \Gamma_{0},
$$

где $f_{j}$ - голоморфная функция из $C^{m}\left(\Gamma_{0}\right)$, равная нулю на $\Gamma_{0} \cap S(R)$ для достаточно малого $R$. Будем искать решение этой краевой задачи в виде $\widetilde{G}_{j}^{\text {in }}(x, k)=$ 
$\widetilde{G}_{j}^{\text {in }}\left(x, k^{*}\right)+\widehat{G}_{j}^{\text {in }}(x, k)$, где $k^{*} \neq k_{0}$ - любое фиксированное число, близкое к $k_{0}$. Тогда краевая задача для $\widehat{G}_{j}^{\text {in }}$ примет вид:

$$
\left(\Delta+k^{2}\right) \widehat{G}_{j}^{\text {in }}=F_{j} \text { при } x \in \Omega, \quad \widehat{G}_{j}^{\text {in }}=0 \text { при } x \in \Gamma_{0},
$$

где $F_{j}$ - голоморфная функция из $C^{m}(\bar{\Omega})$. В силу произвола в выборе $m$ и теорем вложения получаем, что

$$
\widehat{G}_{j}^{\text {in }}(x, k)=\alpha_{j}(k) \frac{\psi(x)}{k^{2}-k_{0}^{2}}+\widehat{g}_{j}^{\text {in }}(x, k),
$$

где $\widehat{g}_{j}^{\text {in }}$ - голоморфная функция из $C^{m}(\bar{\Omega})$, а $\alpha_{j}$ - некоторая голоморфная функция. Следовательно, для производной функции Грина справедливо представление

$$
D_{y}^{j} G^{\text {in }}(x, 0, k)=-\alpha_{j}(0) \frac{\psi(x)}{k_{0}^{2}-k^{2}}+(-1)^{j} \frac{i}{2} D_{x}^{j} \mathscr{H}_{0}^{(1)}(k r)+g_{j}^{\text {in }}(x, k),
$$

где $g_{j}^{\text {in }}(x, k)$ удовлетворяет утверждению леммы.

$\mathrm{C}$ другой стороны, для функции Грина предельной внутренней задачи хорошо известно следующее представление

$$
G^{\text {in }}(x, y, k)=\frac{\psi(x) \psi(y)}{k_{0}^{2}-k^{2}}+\widetilde{g}\left(x, y, k^{2}\right),
$$

где $\widetilde{g}(x, y, \lambda)$ - голоморфная функция из $L_{2}(\Omega)$ при любом фиксированном $y$ в некоторой окрестности точки $\lambda_{0}=k_{0}^{2}$. Применив оператор $D_{y}^{j}$ к вычету (3.3) и сравнив полученный результат в точке $y=0$ с вычетом (3.2), получаем справедливость утверждения леммы для производных функции Грина предельной внутренней задачи.

Для функции Грина предельной внешней задачи из (3.1) следует, что функция $\widetilde{G}_{j}^{\mathrm{ex}}(x, k)=D_{y}^{j} \widetilde{G}^{\mathrm{ex}}(x, 0, k)$ принадлежит $C_{\mathrm{loc}}^{m}\left(\overline{\Omega^{\mathrm{ex}}}\right)$ для любого $m$, удовлетворяет уравнению

$$
\left(\Delta+k^{2}\right) \widetilde{G}_{j}^{\mathrm{ex}}=0 \text { при } x \in \Omega^{\mathrm{ex}}
$$

и граничному условию

$$
\widetilde{G}_{j}^{\mathrm{ex}}=f_{j} \text { при } x \in \Gamma_{0},
$$

где $f_{j}$ - голоморфная функция из $C^{m}\left(\Gamma_{0}\right)$, равная нулю на $\Gamma_{0} \cap S(R)$ для достаточно малого $R$, а при вешественных $k$ функция $\widetilde{G}_{j}^{\text {ex }}(x, k)$ удовлетворяет и условию излучения (1.2). Пусть $L$ - произвольное достаточно большое число такое, что $\bar{\Omega} \subset S(L)$ и $k_{0}^{2}$ не является собственньм значением задачи Дирихле в $\overline{\Omega^{\mathrm{ex}}} \cap S(L)$. Представляя решение краевой задачи $(3.4),(3.5),(1.2)$ и его аналитическое продолжение в виде потенциала простого слоя, получаем, что $\widetilde{G}_{j}^{\text {ex }}$ является решением краевой задачи

$$
\begin{array}{rlrl}
\left(\Delta+k^{2}\right) \widetilde{G}_{j}^{\mathrm{ex}} & =0 & \text { при } & x \in \Omega^{\mathrm{ex}} \cap S(L), \\
\widetilde{G}_{j}^{\mathrm{ex}}=f_{j} & \text { при } & x \in \Gamma_{0}, \\
\widetilde{G}_{j}^{\mathrm{ex}}=f^{(j)} & \text { при } & x \in \partial S(L),
\end{array}
$$


где $f^{(j)}$ - голоморфная функция из $C^{m}(S(L))$ для любого $M$. Применяя к решению краевой задачи (3.6) те же рассуждения, что и вьше для $G_{j}^{\text {in }}$, в силу достаточного произвола в выборе $L$ получаем (с очевидными упрошениями, связанными с голоморфностью решения (3.6) в окрестности точки $k_{0}$ ) справедливость утверждения леммы для производных функции Грина предельной внешней задачи. Лемма доказана.

Так как в окрестности начала координат граница области выпрямлена, то существует $t>0$ такое, что область $\Omega \cap S(t)$ является полукругом в верхней полуплоскости (и, следовательно, $\Omega^{\mathrm{ex}} \cap S(t)$ - полукруг в нижней полуплоскости).

СледСтвиЕ. Нечетное продолжение функции $\left(k_{0}^{2}-k^{2}\right)^{-1} \psi(x) D_{x}^{j} \psi(0)+$ $g_{j}^{\text {in }}(x, k)$ в нижнюю полуплоскость (функиии $g_{j}^{\mathrm{ex}}(x, k)$ в верхнюю полуплоскость) является функиией из $C^{\infty}(\overline{S(t)})$.

ДокАЗАТЕЛЬСтво. Из определения этих функций следует, что они являются решениями уравнения Гельмгольца в $\Omega \cap S(t)$ и $\Omega^{\mathrm{ex}} \cap S(t)$ соответственно и равны нулю на $\partial \Omega \cap S(t)$. Отсюда в силу уравнения и граничных условий вытекает справедливость утверждения следствия.

Обозначим

$$
\begin{gathered}
R_{2}^{(0)}\left(D_{y}\right)=a_{2,0}^{(2)} D_{y}^{2}+a_{2,0}^{(1)} D_{y}^{1}, \quad R_{2}^{(i)}\left(D_{y}\right)=a_{2, i}^{(1)} D_{y}^{1}, \quad i>0 \\
R_{m}^{(n)}\left(D_{y}\right)=\sum_{q=3}^{m} a_{m, n}^{(q)} D_{y}^{q}+a_{m, n}^{(1)} D_{y}^{1}, \quad m \neq 2
\end{gathered}
$$

где $a_{m, i}^{(q)}-$ произвольные постоянные,

$$
\begin{aligned}
& \psi_{\varepsilon}^{\text {in }}(x, k)=\sum_{j=0}^{\infty} \sum_{i=0}^{[j / 2]} \varepsilon^{j-1} \ln ^{i} \varepsilon\left(k_{0}^{2}-k^{2}\right) R_{j+1-2 i}^{(i)}\left(D_{y}\right) G^{\mathrm{in}}(x, 0, k), \\
& \psi_{\varepsilon}^{\mathrm{ex}}(x, k)=\sum_{j=0}^{\infty} \sum_{i=0}^{[j / 2]} \varepsilon^{j-1} \ln ^{i} \varepsilon\left(k^{2}-k_{0}^{2}\right) R_{j+1-2 i}^{(i)}\left(D_{y}\right) G^{\mathrm{ex}}(x, 0, k) .
\end{aligned}
$$

Коэффициенты рядов $\psi_{\varepsilon}^{\text {in }}$ и $\psi_{\varepsilon}^{\text {ex }}$ будем считать продолженными нулем вне $\Omega$ и вне $\Omega^{\text {ex }}$ соответственно. Далее, обозначим через $\psi_{\varepsilon, N}^{\text {in,ex }}$ частичную сумму ряда $\psi_{\varepsilon}^{\text {in,ex }}$ до степеней $\varepsilon^{N}$ включительно. Из леммы 3.1 следует

Лемма 3.2. Функиия

$$
\widetilde{\psi}_{\varepsilon, N}(x, k)=\left(1-\chi\left(r \varepsilon^{-1 / 2}\right)\right)\left(\psi_{\varepsilon, N}^{\text {in }}(x, k)+\psi_{\varepsilon, N}^{\mathrm{ex}}(x, k)\right)
$$

яв.ляется голоморфной функиией из $W_{2,1 о \mathrm{c}}^{1}\left(\mathbb{R}^{2}\right)$ (в некоторой окрестности точки $\left.k_{0}\right)$, удовлетворяет (1.1) при $f=\widetilde{f}_{\varepsilon, N}$, где

$$
\begin{aligned}
\tilde{f}_{\varepsilon, N}(x, k)=- & \left(\psi_{\varepsilon, N}^{\mathrm{in}}(x, k)+\psi_{\varepsilon, N}^{\mathrm{ex}}(x, k)\right) \Delta \chi\left(r \varepsilon^{-1 / 2}\right) \\
& -2 \sum_{i=1}^{2} \frac{\partial}{\partial x_{i}} \chi\left(r \varepsilon^{-1 / 2}\right) \frac{\partial}{\partial x_{i}}\left(\psi_{\varepsilon, N}^{\text {in }}(x, k)+\psi_{\varepsilon, N}^{\mathrm{ex}}(x, k)\right)
\end{aligned}
$$


- голоморфная функиия из $L_{2}\left(\mathbb{R}^{2}\right)$, а ее носитель лежит в $\overline{S\left(2 \varepsilon^{1 / 2}\right)} \backslash$ $S\left(\varepsilon^{1 / 2}\right)$. При вещественных $k$ функция $\widetilde{\psi}_{\varepsilon, N}(x, k)$ удовлетворяет условию излучения (1.2).

Для произвольного $\tau(\varepsilon)=k_{0}+O\left(\varepsilon^{4}\right)$ имеет место следующая сходимость в $W_{2, \text { loc }}^{1}\left(\mathbb{R}^{2}\right)$ :

$$
\widetilde{\psi}_{\varepsilon, N}(x, \tau(\varepsilon)) \underset{\varepsilon \rightarrow 0}{\longrightarrow} a_{2,0}^{(2)} \psi_{2} \psi(x) .
$$

Асимптотики собственной функции $\psi_{\varepsilon}$ будем искать в виде

$$
\begin{array}{ll}
\psi_{\varepsilon}(x)=\psi_{\varepsilon}^{\mathrm{in}}\left(x, \tau_{\varepsilon}\right) & \text { при } x \in \Omega \backslash S\left(\varepsilon^{1 / 2}\right), \\
\psi_{\varepsilon}(x)=\psi_{\varepsilon}^{\mathrm{ex}}\left(x, \tau_{\varepsilon}\right) & \text { при } x \in \Omega^{\mathrm{ex}} \backslash S\left(\varepsilon^{1 / 2}\right), \\
\psi_{\varepsilon}(x)=\sum_{j=2}^{\infty} \sum_{i=0}^{[j / 2]-1} \varepsilon^{j} \ln ^{j} \varepsilon v_{j, i}\left(\frac{x}{\varepsilon}\right) & \text { при } x \in S\left(2 \varepsilon^{1 / 2}\right) .
\end{array}
$$

Краевые задачи для коэффициентов $v_{j, i}(\xi)$ получаются следующим стандартньм образом [11], [14]. В (1.1) при $f=0$ подставляем ряды (2.1) и (3.9) вместо $k$ и $E_{\varepsilon}$ соответственно, делаем замену переменных $x=\xi \varepsilon$ и выписываем отдельно равенства при одинаковых степенях $\varepsilon$ и $\ln \varepsilon$. Затем переходим к формальному пределу при $\varepsilon \rightarrow 0$. В итоге получаем следуюшую рекуррентную систему краевых задач:

$$
\begin{array}{rlrl}
\Delta_{\xi} v_{j, i} & =-\sum_{p=4}^{j-4} \sum_{q=0}^{[p / 2]-2} \lambda_{p, q} v_{j-p-2, i-q} \lambda_{0} v_{j-2, i} & \text { при } \xi \in \mathbb{R}^{2} \backslash \bar{\gamma}, \\
v_{j, i}=0 & \text { при } \xi \in \gamma,
\end{array}
$$

где $\lambda_{0}=k_{0}^{2}$ и $\lambda_{p, q}-$ коэффициенты ряда $\lambda_{\varepsilon}=\tau_{\varepsilon}^{2}$. Обозначим через $V_{\varepsilon, N}(\xi)$ частичную сумму ряда (3.9).

Лемма 3.3. Пусть функиии $v_{j, i}(\xi) \in W_{2, \text { loc }}^{1}\left(\mathbb{R}^{2}\right)$ имеют асимптотики $O\left(\rho^{j-2 i}\right)$ при $\rho=|\xi| \rightarrow \infty$ и являются решениями рекуррентной системы краевых задач (3.10). Тогда функиия

$$
\widehat{\psi}_{\varepsilon, N}(x)=\chi\left(r \varepsilon^{-1 / 2}\right) V_{\varepsilon, N}\left(\frac{x}{\varepsilon}\right)
$$

имеет норму $о\left(\varepsilon^{1 / 2}\right)$ в $W_{2}^{1}\left(\mathbb{R}^{2}\right)$, является решением (1.1), (1.2) при $f(x)=$ $\widehat{f}_{\varepsilon, N}(x, k)$, где $\widehat{f}_{\varepsilon, N}$ - голоморфная функция из $L_{2}\left(\mathbb{R}^{2}\right)$, а ее носитель лежит ${ }_{\boldsymbol{B}} \overline{S\left(2 \varepsilon^{1 / 2}\right)}$.

Для функиии $\tau(\varepsilon)$, имеющей асимптотику (2.1), справедливо представленuе

$\widehat{f}_{\varepsilon, N}(x, \tau(\varepsilon))=V_{\varepsilon, N}\left(\frac{x}{\varepsilon}\right) \Delta \chi\left(r \varepsilon^{-1 / 2}\right)+2 \sum_{i=1}^{2} \frac{\partial}{\partial x_{i}} \chi\left(r \varepsilon^{-1 / 2}\right) \frac{\partial}{\partial x_{i}} V_{\varepsilon, N}\left(\frac{x}{\varepsilon}\right)+\widehat{f}_{\varepsilon}^{(N)}(x)$,

где функиия $\widehat{f}_{\varepsilon}^{(N)}(x)=\chi\left(r \varepsilon^{-1 / 2}\right)\left(\Delta+r^{2}(\varepsilon)\right) V_{\varepsilon, N}\left(x \varepsilon^{-1}\right)$ имеет норму $O\left(\varepsilon^{N / 2}\right)$ в $L_{2}\left(\mathbb{R}^{2}\right)$. 
ДокАЗАТЕльство. Справедливость утверждения леммы для $\widehat{f}_{\varepsilon}^{(N)}$ следует в силу равенств (3.10) и условия леммы на порядки роста на бесконечности функций $v_{j, i}(\xi)$. Справедливость остальных утверждений леммы очевидна.

Из утверждений лемм 3.2 и 3.3 вытекает

ЛЕмма 3.4. Пусть выполнены условия леммы 3.3. Тогда функиия

$$
\psi_{\varepsilon, N}(x, k)=\widetilde{\psi}_{\varepsilon, N}(x, k)+\widehat{\psi}_{\varepsilon, N}(x)
$$

является голоморфной функиией из $W_{2, \mathrm{loc}}^{1}\left(\mathbb{R}^{2}\right)$ (в некоторой окрестности точки $k_{0}$ ), удовлетворяет (1.1) при $f=f_{\varepsilon, N}$, әде $f_{\varepsilon, N}$ - голоморфная функиия из $L_{2}\left(\mathbb{R}^{2}\right)$, а ее носитель лежит в $\overline{S\left(2 \varepsilon^{1 / 2}\right)}$. При вещественных $k$ функиия $\psi_{\varepsilon, N}(x, k)$ удовлетворяет условию излучения (1.2). Имеет место следующая сходимость в $W_{2,1 \text { ос }}^{1}\left(\mathbb{R}^{2}\right)$ :

$$
\psi_{\varepsilon, N}(x, \tau(\varepsilon)) \underset{\varepsilon \rightarrow 0}{\longrightarrow} a_{2,0}^{(2)} \psi_{2} \psi(x) .
$$

Если к тому жсе функиия

$$
\begin{aligned}
& \left(V_{\varepsilon, N}\left(\frac{x}{\varepsilon}\right)-\psi_{\varepsilon, N}^{\mathrm{in}}(x, \tau(\varepsilon))-\psi_{\varepsilon, N}^{\mathrm{ex}}(x, \tau(\varepsilon))\right) \Delta \chi\left(r \varepsilon^{-1 / 2}\right) \\
& \quad+2 \sum_{i=1}^{2} \frac{\partial}{\partial x_{i}} \chi\left(r \varepsilon^{-1 / 2}\right) \frac{\partial}{\partial x_{i}}\left(V_{\varepsilon, N}\left(\frac{x}{\varepsilon}\right)-\psi_{\varepsilon, N}^{\mathrm{in}}(x, \tau(\varepsilon))-\psi_{\varepsilon, N}^{\mathrm{ex}}(x, \tau(\varepsilon))\right)
\end{aligned}
$$

имеет норму $O\left(\varepsilon^{N_{1}}\right)$ в $L_{2}\left(\mathbb{R}^{2}\right)$, әде $N_{1}$ растет неограниченно вместе $c N$, то функиия $f_{\varepsilon, N}(x, \tau(\varepsilon))$ имеет норму $O\left(\varepsilon^{N_{2}}\right)$ в $L_{2}\left(\mathbb{R}^{2}\right)$, где $N_{2}=\min \left\{N / 2 ; N_{1}\right\}$.

Таким образом, для построения формального асимптотического разложения полюса и соответствующей собственной функции достаточно построить ряды (2.1), (3.7)-(3.9), удовлетворяющие условиям леммы 3.4, что будет достигаться согласованием рядов (3.7)-(3.9).

Через $P_{j}(\xi)$ обозначим однородные полиномы степени $j$, тождественно равные нулю при $\xi_{2}=0$, а через $H_{q}(\xi)$ - однородные функции степени $q$, представимые в виде $H_{q}(\xi)=P_{q+2 j}(\xi) \rho^{-2 j}$ при каком-нибудь целом $j \geqslant 0$.

Пусть $\widetilde{\mathscr{B}}_{j}-$ множество рядов вида

$$
H(\xi)=\sum_{q=-j}^{\infty} H_{q}(\xi)+\ln \rho \sum_{q=1}^{j} P_{q}(\xi)
$$

Ряды $H^{(1)}(\xi)$ и $H^{(2)}(\xi)$ будем назьвать сопряжсенными, если разность $H^{(1)}(\xi)-$ $H^{(2)}\left(\xi_{*}\right)$ является многочленом.

На суммах $U(x, \varepsilon)$ вида $\psi_{\varepsilon}^{\text {in, } е}(x, \tau(\varepsilon))$ определим оператор $K_{N}$ следующим образом [11], [14]. Коэффициенты ряда $U(x, \varepsilon)$ заменяем на их асимптотики при $r \rightarrow 0$, а функцию $r(\varepsilon)$ - на ее асимптотику и переходим к переменным $\xi=x \varepsilon^{-1}$. В полученном двойном ряду оставляем члены вида $\varepsilon^{j} \ln ^{i} \varepsilon \Phi(\xi)$ при $j \leqslant N$. Эту сумму и будем обозначать $K_{N}(U(x, \varepsilon))$. 
ЗАмечАниЕ 3.1 . Очевидно, функция $\operatorname{Im} G_{q}^{\mathrm{ex}}\left(x, 0, k_{0}\right)$ удовлетворяет уравне-

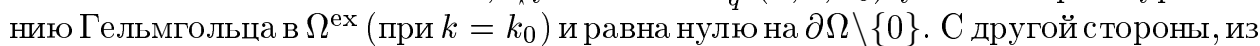
леммы 3.1 следует, что $\operatorname{Im} G_{q}^{\mathrm{ex}}\left(x, 0, k_{0}\right) \in C^{\infty}\left(\overline{\Omega^{\mathrm{ex}} \cap S(R)}\right)$ для достаточно малого $R>0$ и, следовательно, эта функция равна нулю на $\partial \Omega$. Отсюда в силу уравнения и граничных условий получаем, что

$$
\operatorname{Im} G_{q}^{\mathrm{ex}}\left(x, 0, k_{0}\right)=g_{q, 1} x_{1}+g_{q, 2} x_{1} x_{2}+O\left(r^{3}\right) \text { при } r \rightarrow 0 .
$$

Обозначим через $\theta$ полярный угол. С учетом замечания 3.1 из леммы 3.1 и ее следствия вытекает

Лемма 3.5. Пусть асимптотика некоторой функиии $\tau(\varepsilon)$ имеет вид (2.1). Тогда для любого целого $N \geqslant 2$ справедливы равенства

$$
K_{N}\left(\psi_{\varepsilon}^{\mathrm{in}, \mathrm{ex}}\left(x, \tau_{\varepsilon}\right)\right)=\sum_{j=2}^{N} \sum_{i=0}^{[j / 2]-1} \varepsilon^{j} \ln ^{i} \varepsilon V_{j, i}^{\mathrm{in}, \mathrm{ex}}(\xi)
$$

где ряды $V_{j, q}^{\mathrm{in}, \mathrm{ex}}$ сопряжень при любых фиксированных $j$ и $q$.

Рядыц $V_{j, 0}^{\mathrm{in}, \mathrm{ex}} \in \widetilde{\mathscr{B}}_{j}, V_{j, i}^{\mathrm{in}, \mathrm{ex}} \in \widetilde{\mathscr{B}}_{j-2 i-2}($ при $i>0)$ и являются формальньми асимптотическими решениями (3.10) при $\rho \rightarrow \infty$, әде в правой части уравнения функиии $v_{j, i}$ заменены на ряды $V_{j, i}^{\mathrm{in}, \mathrm{ex}}$.

Справедливы представления:

$$
\begin{aligned}
V_{2,0}^{\text {in }, \mathrm{ex}}(\xi) & =\widetilde{V}_{2,0}^{\mathrm{in}, \mathrm{ex}}(\xi) \mp \frac{2 k_{0}}{\pi} \tau_{4,0} \Pi_{0,0}, \\
V_{j, i}^{\mathrm{in}, \mathrm{ex}}(\xi) & =\widetilde{V}_{j, i}^{\mathrm{in}, \mathrm{ex}}(\xi) \mp \frac{2 k_{0}}{\pi}\left(\tau_{j+2, i} \Pi_{0}+\tau_{4,0} \Pi_{j-2, i}\right) \quad n p u \quad j>2, \\
\Pi_{n, i} & =\sum_{q=1}^{\infty}(q-1) ! a_{n+q, i}^{(q)} \rho^{-q} \sin (q \theta), \\
\widetilde{V}_{2,0}^{\mathrm{in}}(\xi) & =\frac{1}{2} a_{2,0}^{(2)} \psi_{2}^{2} \rho^{2} \sin (2 \theta), \quad \widetilde{V}_{2,0}^{\mathrm{ex}}(\xi)=\widetilde{V}_{3,0}^{\mathrm{ex}}(\xi) \equiv 0, \\
\widetilde{V}_{3,0}^{\mathrm{in}}(\xi) & =\frac{1}{6} \psi_{2} \psi_{3}\left(a_{2,0}^{(2)} \rho^{3} \sin (3 \theta)+3 a_{3,0}^{(3)} \rho^{2} \sin (2 \theta)\right), \\
\widetilde{V}_{4,1}^{\mathrm{in}, \mathrm{ex}}(\xi) & = \pm \pi^{-1} k_{0}^{3} \tau_{4,0} a_{1,0}^{(1)} \rho \sin \theta
\end{aligned}
$$

где рядьь $\widetilde{V}_{j, i}^{\mathrm{in}, \mathrm{ex}}$ не зависят от $\tau_{m+2, k}$ и $a_{m+q-2, k}^{(q)}$ nрu $m \geqslant j$.

Eслик тому же $\operatorname{Im} \tau_{4,0}=\operatorname{Im} a_{j, 0}^{(j)}=\operatorname{Im} a_{4,0}^{(3)}=\operatorname{Im} \tau_{4,0}=0, m o$

$$
\operatorname{Im} \widetilde{V}_{4,0}^{\mathrm{in}}(\xi)=0, \quad \operatorname{Im} \widetilde{V}_{4,0}^{\mathrm{ex}}(\xi)=2 k_{0} \tau_{4,0} a_{1,0}^{(1)}\left(\left.\frac{\partial}{\partial x_{2}} \operatorname{Im} G_{1}^{\mathrm{ex}}\left(x, 0, k_{0}\right)\right|_{x=0}\right) \rho \sin \theta
$$

Обозначим через $\mathscr{B}$ j множество функций $v \in W_{2, \text { lос }}^{1}\left(\mathbb{R}^{2}\right) \cap C^{\infty}\left(\mathbb{R}^{2} \backslash \bar{\gamma}\right)$ таких, что они равны нулю на $\gamma$, имеют при $\rho \rightarrow \infty$ дифференцируемые асимптотики из $\widetilde{\mathscr{B}}_{j}$ (вообше говоря, разные при $\xi_{2}>0$ и $\xi_{2}<0$ ), а разность $v(\xi)-v\left(\xi_{*}\right)$ является многочленом. 
Заметим, что ранее определенные гармонические функции $Y_{1}$ и $Y_{2}$ принадлежат $\mathscr{B}_{1}$ и $\mathscr{B}_{2}$ соответственно и имеют следуюшие асимптотики при $\rho \rightarrow \infty:$

$$
\begin{array}{ll}
Y_{q}(\xi)=\rho^{q} \sin (q \theta)+\sum_{j=1}^{\infty} c_{q, j} \rho^{-j} \sin (j \theta) & \text { при } \xi_{2}>0, \\
Y_{q}(\xi)=-\sum_{j=1}^{\infty} c_{q, j} \rho^{-j} \sin (j \theta) & \text { при } \xi_{2}<0 .
\end{array}
$$

В дальнейшем (в $\S 4)$ нам понадобятся гармонические функции $Y_{3} \in \mathscr{B}_{3}$ и $Y_{4} \in \mathscr{B}_{4}$, имеющие на бесконечности указанные выше асимптотики. Получить явные представления для функций $Y_{q}$ (в том числе и для $\left.q=1,2\right)$ достаточно просто следующим образом. Вначале нужно рассмотреть случай $\omega_{+}=-\omega_{-}=1$. Легко видеть, что в этом случае функции $Y_{q}=\widehat{Y}_{q}$, имеющие заданный вид асимптотик на бесконечности, представимы в виде

$$
\begin{aligned}
\widehat{Y}_{j}(\xi) & =\frac{1}{2} \operatorname{Im}\left(\zeta^{j}+\zeta^{j-1}\left(\zeta^{2}-1\right)^{1 / 2}\right) & \text { при } j=1,2, \\
\widehat{Y}_{j}(\xi) & =\frac{1}{2} \operatorname{Im}\left(\zeta^{j}+\zeta^{j-1}\left(\zeta^{2}-1\right)^{1 / 2}\right)+\frac{1}{4}\left(\widehat{Y}_{j-2}(\xi)+\widehat{Y}_{j-2}\left(\xi_{*}\right)\right) & \text { при } j=3,4 .
\end{aligned}
$$

Далее, используя растяжение системы координат с коэффициентом $t=\frac{1}{2}\left(\omega_{+}-\omega_{-}\right)$ и сдвиг ее вдоль оси $O \xi_{1}$ на $\varkappa_{\omega}$, последовательно получаем искомые функции в виде

$$
\begin{aligned}
& Y_{1}(\xi)=t \widehat{Y}_{1}\left(\frac{\xi_{1}-\varkappa_{\omega}}{t}, \frac{\xi_{2}}{t}\right), \\
& Y_{2}(\xi)=t^{2} \widehat{Y}_{2}\left(\frac{\xi_{1}-\varkappa_{\omega}}{t}, \frac{\xi_{2}}{t}\right)+2 \varkappa_{\omega} Y_{1}(\xi), \\
& Y_{3}(\xi)=t^{3} \widehat{Y}_{3}\left(\frac{\xi_{1}-\varkappa_{\omega}}{t}, \frac{\xi_{2}}{t}\right)+3 \varkappa_{\omega} Y_{2}(\xi)-3 \varkappa_{\omega}^{2} Y_{1}(\xi), \\
& Y_{4}(\xi)=t^{4} \widehat{Y}_{4}\left(\frac{\xi_{1}-\varkappa_{\omega}}{t}, \frac{\xi_{2}}{t}\right)+4 \varkappa_{\omega} Y_{3}(\xi)-6 \varkappa_{\omega}^{2} Y_{2}(\xi)+4 \varkappa_{\omega}^{3} Y_{1}(\xi) .
\end{aligned}
$$

Принимая во внимание определение функций $\widehat{Y}_{q}$ и равенство $4 c_{\omega}=t^{2}$, получаем для функций $Y_{1}$ и $Y_{2}$ вид, приведенньй в $\S 2$, а для функций $Y_{3}$ и $Y_{4}-$ вид

$$
\begin{aligned}
Y_{3}(\xi)= & \frac{1}{2} \operatorname{Im}\left(\zeta^{3}+\zeta^{2}\left(\left(\zeta-\omega_{-}\right)\left(\zeta-\omega_{+}\right)\right)^{1 / 2}\right)+c_{\omega}\left(Y_{1}(\xi)+Y_{1}\left(\xi_{*}\right)\right) \\
& +2 \varkappa_{\omega} Y_{2}(\xi)-\varkappa_{\omega}^{2} Y_{1}(\xi) \\
Y_{4}(\xi)= & \frac{1}{2} \operatorname{Im}\left(\zeta^{4}+\zeta^{3}\left(\left(\zeta-\omega_{-}\right)\left(\zeta-\omega_{+}\right)\right)^{1 / 2}\right)+c_{\omega}\left(Y_{2}(\xi)+Y_{2}\left(\xi_{*}\right)\right) \\
& -2 c_{\omega} \varkappa\left(Y_{1}(\xi)+Y_{1}\left(\xi_{*}\right)\right)+4 \varkappa_{\omega} Y_{3}(\xi)-6 \varkappa_{\omega}^{2} Y_{2}(\xi)+4 \varkappa_{\omega}^{3} Y_{1}(\xi) .
\end{aligned}
$$

На этом же пути вычисляются и значения коэффициентов $c_{i, j}$ и, в частности, получается, что

$$
\begin{array}{rlrl}
c_{1,1} & =c_{\omega}, \\
c_{3,1} & =3 c_{1,3}=3 c_{\omega}\left(c_{\omega}+\varkappa_{\omega}^{2}\right), & & c_{2,1}=2 c_{1,2}=2 c_{\omega} \varkappa_{\omega}, \\
2 c_{3,2} & =3 c_{2,3}=6 \varkappa_{\omega} c_{\omega}\left(2 c_{\omega}+\varkappa_{\omega}^{2}\right), & & c_{2,2}=c_{\omega}\left(c_{\omega}+2 \varkappa_{\omega}^{2}\right), \\
c_{3,3} & =c_{\omega}\left(4 c_{\omega}^{2}+12 c_{\omega} \varkappa_{\omega}^{2}+\varkappa_{\omega}^{4}\right) . & &
\end{array}
$$


Лемма 3.6. Пусть функиия $F \in \mathscr{B}_{j-2}$, а сопрязсенные ряды $V^{ \pm} \in \widetilde{\mathscr{B}}_{j}$ являются формальными асимптотическими решениями уравнения $\Delta V^{ \pm}=F$ при $\rho \rightarrow \infty, \pm \xi_{2}>0$. Тогда существует функиия $v \in \mathscr{B}_{j}$, являющаяся решением краевой задачи

$$
\Delta v=F \quad n p u \quad \xi \notin \bar{\gamma}, \quad v=0 \quad n p u \quad \xi \in \gamma
$$

и имеющая при $\rho \rightarrow \infty$ асимптотики

$$
v(\xi)=V^{ \pm}(\xi) \pm \sum_{j=1}^{\infty} c_{j}^{ \pm} \rho^{-j} \sin (j \theta) \quad n p u \quad \pm \xi_{2} \geqslant 0
$$

əде $c_{j}^{+}=c_{j}^{-}=c_{j}$.

ДокАЗАтЕльство. Обозначим через $F_{ \pm}$сужения функции $F$ на полуплоскости $\mathbb{R}_{ \pm}^{2}=\left\{\xi: \pm \xi_{2}>0\right\}$. Стандартньм вычитанием растущих на бесконечности членов и использованием явных формул для решения задачи Дирихле в полуплоскости легко показать (см., например, [14]), что сушествуют решения $v^{ \pm} \in$ $W_{2, \text { loc }}^{2}\left(\mathbb{R}_{ \pm}^{2}\right)$ задач Дирихле для уравнений $\Delta v^{ \pm}=F_{ \pm}$в $\mathbb{R}_{ \pm}^{2}$, имеющие при $\rho \rightarrow \infty$ асимптотики (3.12) при некоторых постоянных $c_{q}^{ \pm}$. Так как $F \in \mathscr{B}_{j-2}$, то функция $v^{+}(\xi)-v\left(\xi_{*}\right)$ является решением задачи Дирихле в $\mathbb{R}_{+}^{2}$ с правой частью, равной многочлену. Следовательно, функция $v^{+}(\xi)-v\left(\xi_{*}\right)$ также является многочленом. А так как $V^{ \pm} \in \widetilde{\mathscr{B}}_{j}$, то отсюда следует, что $c^{+}=c^{-}$(в асимптотиках функций $\left.v^{ \pm}(\xi)\right)$.

Обозначим $\omega=\left\{\xi=\left(\xi_{1}, 0\right): \omega_{-}<\xi_{1}<\omega_{+}\right\}$. Известно (см., например, [16]), что существует гармоническая вне $\bar{\omega}$ функция $V \in W_{2, \text { lос }}^{1}\left(\mathbb{R}^{2} \backslash \bar{\omega}\right)$, представимая в виде потенциала двойного слоя и удовлетворяющая граничному условию

$$
\frac{\partial}{\partial \xi_{2}} V=\phi, \quad \xi \in \omega
$$

для $\phi \in L_{2}(\omega)$. Положим

$$
\phi=\frac{\partial}{\partial \xi_{2}}\left(v^{+}-v^{-}\right)
$$

Легко видеть, что функция

$$
v=v^{+}-\frac{1}{2} V, \quad \xi_{2} \geqslant 0, \quad v=v^{-}+\frac{1}{2} V, \quad \xi_{2} \leqslant 0,
$$

удовлетворяет утверждению леммы.

СлЕДСТВИЕ 1. Если $v^{(1)} u v^{(2)}-$ два решения (3.11) из $\mathscr{B}_{j} u v^{(1)}(\xi)-$ $v^{(2)}(\xi)=O\left(\rho^{-1}\right)$ nрu $\rho \rightarrow \infty$, mo $v^{(1)}=v^{(2)}$.

Справедливость этого утверждения легко показать, интегрируя по частям правую часть равенства

$$
0=\int_{S(R)}\left(v^{(1)}-v^{(2)}\right) \Delta\left(v^{(1)}-v^{(2)}\right) d \xi
$$

при $R \rightarrow \infty$. 
СлЕДСТВИЕ 2. Если $\omega_{-}=-\omega_{+}$, а функиия $F$ и ряды $V^{ \pm}$четные (нечетнье) по переменной $\xi_{1}$, то функция $v$ является тоже четной (нечетной) по этой переменной, а постояннье $c_{2 j}=0 \quad\left(c_{2 j+1}=0\right)$.

ЗАмечание 3.2. Очевидно, что если функция $F$ и ряды $V^{ \pm}$вешественные, то функция $v$ и постоянные $c_{q}$ также вешественные.

Леммы 3.5 и 3.6 позволяют перейти к согласованию рядов (3.7)-(3.9).

ТеОРема 3.1. Пусть $\omega_{-} \neq-\omega_{+}, N \geqslant 2$ - произвольное челое число. Тогда существуют функиия $\tau(\varepsilon)$, имеющая асимптотику вида (2.1), и ряды $\psi_{\varepsilon}^{\text {in,ex }}(x, k)$ u (3.9) такие, что коэффичиенты $v_{j, 0} \in \mathscr{B}_{j}, v_{j, i} \in \mathscr{B}_{j-2 i-2}$ (при $i>0$ ) являются решениями краевых задач (3.11), для любого $N \geqslant 2$ справедливы равенства

$$
V_{\varepsilon, N}(\xi)=K_{N}\left(\psi_{\varepsilon}^{\text {in }, \mathrm{ex}}(x, \tau(\varepsilon))\right) \quad n p u \quad \rho \rightarrow \infty, \quad \pm \xi_{2} \geqslant 0,
$$

коэффициенты $\tau_{4,0}, \tau_{5,0}, \tau_{6,1}, \tau_{6,0}$ удовлетворяют соотночениям (2.2) и

$$
a_{1,0}^{(1)}=\psi_{2}^{-1} c_{2,2}^{-1} c_{2,1}, \quad a_{2,0}^{(2)}=\psi_{2}^{-1}, \quad v_{2,0}=\frac{1}{2} \psi_{2} Y_{2} .
$$

ДоКАЗАТЕЛЬСТво. Выбрав $a_{2,0}^{(2)}$ в соответствии с (3.14), в силу леммы 3.4 получаем, что для любой функции $\tau(\varepsilon)$, имеющей асимптотику вида $(2.1)$, имеет место сходимость

$$
\psi_{\varepsilon, N}(x, \tau(\varepsilon)) \underset{\varepsilon \rightarrow 0}{\longrightarrow} \psi(x)
$$

в $W_{2, \text { loc }}^{1}\left(\mathbb{R}^{2}\right)$.

По определению функция

$$
v_{2,0}(\xi)=\frac{1}{2} a_{2,0}^{(2)} \psi_{2}^{2} Y_{2}(\xi)
$$

является решением краевой задачи (3.10), и в силу леммы 3.5 для ее асимптотик на бесконечности справедливы равенства

$$
\begin{aligned}
v_{2,0}(\xi)-V_{2,0}^{\text {in }, \text { ex }}(\xi)= & \pm\left(\frac{1}{2} a_{2,0}^{(2)} \psi_{2}^{2} \sum_{q=1}^{\infty} c_{2, q} \rho^{-q} \sin (q \theta)\right. \\
& \left.+\tau_{4,0} \frac{2 k_{0}}{\pi} \sum_{q=1}^{\infty} \sum_{q=1}^{\infty}(q-1) ! a_{q, 0}^{(q)} \rho^{-q} \sin (q \theta)\right) \quad \text { при } \quad \pm \xi_{2} \geqslant 0 .
\end{aligned}
$$

Приравнивая в последнем равенстве коэффициенты при $\rho^{-2}$, получаем, что

$$
\tau_{4,0}=-\frac{\pi}{4 k_{0}} \psi_{2}^{2} c_{2,2}
$$

Подставляя в (3.17) значение $c_{2,2}$, получаем значение $(2.2)$ для $\tau_{4,0}$. Приравнивая же коэффициенты при остальных степенях $\rho$, определяем постоянные $a_{q, 0}^{(q)}$ для 
$q \neq 2$ и, в частности, принимая во внимание $(3.17)$ и то, что $a_{2,0}^{(2)}=\psi^{-1}$, получаем значение (3.14) для $a_{1,0}^{(1)}$, а также равенство

$$
a_{3,0}^{(3)}=\left(2 \psi_{2} c_{2,2}\right)^{-1} c_{2,3}
$$

И, наконец, подставляя значение $(3.14)$ для $a_{2,0}^{(2)}$ в $(3.16)$, получаем $(3.14)$ для $v_{2,0}$. В результате проделанного согласования построены постоянные $\tau_{4,0}, a_{q, 0}^{(q)}$ и функция $v_{2,0}$ такие, что оказалось выполненным равенство (3.13) для $N=2$.

Дальнейшее доказательство проводится по индукции. Допустим, что при $j \leqslant M$ существуют постоянные $a_{q+j-2, i}^{(q)}, \tau_{j+2, i}$ и функции $v_{j, 0} \in \mathscr{B}_{j}, v_{j, i} \in \mathscr{B}_{j-2 i-2}$ (при $i>0$ ), являющиеся решениями краевых задач $(3.10)$, такие, что при $\rho \rightarrow \infty$ имеют место равенства (3.13). Тогда в силу лемм 3.5, 3.6 при $0 \leqslant i \leqslant[(M+1) / 2]-1$ сушествуют функции $v_{M+1,0} \in \mathscr{B}_{M+1}, v_{M+1, i} \in \mathscr{H}_{M-2 i-1}$ (при $i>0)$, являющиеся решениями краевых задач $(3.10)$, такие, что при $\rho \rightarrow \infty$ имеют место равенства

$$
v_{M+1, i}(\xi)=\widetilde{V}_{M+1, i}^{\mathrm{in}, \mathrm{ex}}(\xi) \pm \sum_{j=1}^{\infty} c_{j} \rho^{-j} \sin (j \theta), \quad \pm \xi_{2} \geqslant 0
$$

Приравнивая правую часть $(3.19)$ к $V_{M+1, i}^{\text {in,ex }}(\xi)$, последовательно находим постоянные $\tau_{M+3, i}$ и $a_{q+M-1, i}^{(q)}$ и получаем асимптотическое равенство при $\rho \rightarrow \infty$ :

$$
v_{M+1, i}(\xi)=V_{M+1, i}^{\text {in }, \text { ex }}(\xi), \quad \pm \xi_{2} \geqslant 0
$$

Из равенства (3.20) и справедливости равенства (3.13) при $N=M$ следует справедливость равенства (3.13) при $N=M+1$.

Покажем справедливость остальных равенств в (2.1). Формула для $\tau_{5,0}$ получается из следующей цепочки (см. лемму 3.5 , лемму 3.6 , ее следствие 1 , равенства $(3.14),(3.18)$ и асимптотики функций $\left.Y_{q}(\xi)\right)$ :

$$
\begin{aligned}
\widetilde{V}_{3,0}^{\mathrm{ex}}(\xi) & =0 \\
\widetilde{V}_{3,0}^{\mathrm{in}}(\xi) & =\frac{1}{6} a_{2,0}^{(2)} \psi_{2} \psi_{3} \rho^{3} \sin (3 \theta)+\frac{1}{2} a_{3,0}^{(3)} \psi_{3} \psi_{2} \rho^{2} \sin (2 \theta) \\
& \Rightarrow v_{3,0}=\psi_{3}\left(\frac{1}{6} Y_{3}+\frac{1}{4} c_{2,3} c_{2,2}^{-1} Y_{2}\right) \\
& \Rightarrow \psi_{3}\left(\frac{1}{6} c_{3,2}+\frac{1}{4} c_{2,3} c_{2,2}^{-1} c_{2,2}\right)=-\frac{2 k_{0}}{\pi} \tau_{5,0} \psi_{2}^{-1} \\
& \Rightarrow \tau_{5,0}=-\frac{\pi}{2 k_{0}} \psi_{2} \psi_{3}\left(\frac{1}{3} c_{3,2}+\frac{1}{2} c_{2,3}\right) .
\end{aligned}
$$

Подставляя в последнее равенство значения $c_{j, q}$, получаем соотношение $(2.2)$ для $\tau_{5,0}$. Приравнивая $V_{3,0}^{\mathrm{in}, \mathrm{ex}}$ и асимптотики $v_{3,0}$, легко убедиться, что $\operatorname{Im} a_{4,0}^{(3)}=0$. 
Значение $\tau_{6,1}$ находится (с учетом (3.14), следствия 1 леммы 3.6) по следуюшей цепочке:

$$
\begin{aligned}
\widetilde{V}_{4,1}^{\text {in }, \mathrm{ex}}(\xi) & = \pm \pi^{-1} k_{0}^{3} \tau_{4,0} a_{1,0}^{(1)} \rho \sin \theta \\
& \Rightarrow v_{4,1}(\xi)=\pi^{-1} k_{0}^{3} \tau_{4,0} a_{1,0}^{(1)}\left(Y_{1}(\xi)+Y_{1}\left(\xi_{*}\right)\right) \\
& \Rightarrow 2 \pi^{-1} k_{0}^{3} \tau_{4,0} \psi_{2}^{-1} c_{2,2}^{-1} c_{2,1} c_{1,2}=-\frac{2 k_{0}}{\pi} \tau_{6,1} \psi_{2}^{-1} \\
& \Rightarrow \tau_{6,1}=-k_{0}^{2} \tau_{4,0} c_{2,2}^{-1} c_{2,1} c_{1,2}
\end{aligned}
$$

Подставляя в последнее равенство значения $\tau_{4,0}$ и $c_{j, q}$, получаем соотношение $(2.2)$ для $\tau_{6,1}$.

И, наконец, значение $\operatorname{Im} \tau_{6,0}$ определяем по цепочке (с учетом $(3.14)$, леммы 3.5 , следствия 1 леммы 3.6 и замечания 3.2 ):

$$
\begin{aligned}
\operatorname{Im} \widetilde{V}_{4,0}^{\mathrm{in}}(\xi) & =0 \\
\operatorname{Im} \widetilde{V}_{4,0}^{\mathrm{ex}}(\xi) & =2 k_{0} \tau_{4,0} a_{1,0}^{(1)}\left(\left.\frac{\partial \operatorname{Im} G_{1}^{\mathrm{ex}}\left(x, 0, k_{0}\right)}{\partial x_{2}}\right|_{x=0}\right) \rho \sin \theta \\
& \Rightarrow \operatorname{Im} v_{4,0}(\xi)=-2 k_{0} \tau_{4,0} a_{1,0}^{(1)}\left(\left.\frac{\partial \operatorname{Im} G_{1}^{\mathrm{ex}}\left(x, 0, k_{0}\right)}{\partial x_{2}}\right|_{x=0}\right) Y_{1}\left(\xi_{*}\right) \\
\Rightarrow & -2 k_{0} \tau_{4,0} \psi_{2}^{-1} c_{2,2}^{-1} c_{2,1}\left(\left.\frac{\partial \operatorname{Im} G_{1}^{\mathrm{ex}}\left(x, 0, k_{0}\right)}{\partial x_{2}}\right|_{x=0}\right) c_{1,2} \\
& =-\frac{2 k_{0}}{\pi} \psi_{2}^{-1} \operatorname{Im} \tau_{6,0} \\
\Rightarrow & \operatorname{Im} \tau_{6,0}=\pi \tau_{4,0} c_{2,2}^{-1} c_{2,1} c_{1,2}\left(\left.\frac{\partial \operatorname{Im} G_{1}^{\mathrm{ex}}\left(x, 0, k_{0}\right)}{\partial x_{2}}\right|_{x=0}\right)
\end{aligned}
$$

Интегрируя по частям левую часть тождества

$$
\operatorname{Im} \int_{B(R)} \overline{G_{1}^{\mathrm{ex}}\left(x, 0, k_{0}\right)}\left(\Delta+k_{0}^{2}\right) G_{1}^{\mathrm{ex}}\left(x, 0, k_{0}\right) d s \equiv 0
$$

где $B(R)=S(R) \backslash\left(S\left(R^{-1}\right) \cup \Omega\right)$, и переходя к пределу при $R \rightarrow \infty$, получаем равенство

$$
\left(\left.\frac{\partial \operatorname{Im} G_{1}^{\mathrm{ex}}\left(x, 0, k_{0}\right)}{\partial x_{2}}\right|_{x=0}\right)=k_{0} \sigma_{1,1}
$$

Подставляя (3.22) и значения $\tau_{4,0}$ и $c_{j, q}$ в $(3.21)$, получаем соотношение $(2.2)$ для $\operatorname{Im} \tau_{6,0}$. Теорема доказана.

ДоКАЗАТЕЛЬСтво ТЕОРЕмы 2.1. Из определения $\psi_{\varepsilon}^{\text {in }}, \psi_{\varepsilon}^{\text {ex }}$, оператора $K_{N}$ и из леммы 3.1 следует, что для любой функции $\tau(\varepsilon)$, имеющей асимптотику вида $(2.1)$, справедливо равенство

$$
\begin{gathered}
\psi_{\varepsilon, N}^{\text {in }, \mathrm{ex}}(x, \tau(\varepsilon))-K_{N}\left(\psi_{\varepsilon, N}^{\mathrm{in}, \mathrm{ex}}(x, \tau(\varepsilon))\right)=O\left(r^{N+1}+\varepsilon^{3} r^{N-2}\right) \\
\text { при } \varepsilon^{1 / 2}<r<2 \varepsilon^{1 / 2}, \quad \pm x_{2}>0
\end{gathered}
$$

Причем это равенство можно дифференцировать (по переменньм $x_{j}$ ). 
Пусть ряды $\psi_{\varepsilon}^{\text {in }}, \psi_{\varepsilon}^{\text {ex }}$ и $(3.9)$ и функция $\tau(\varepsilon)$ удовлетворяют утверждениям теоремы 3.1. Тогда, во-первых, выполнены условия леммы 3.3. Покажем, что и оставшееся условие леммы 3.4 выполнено. В силу леммы 3.1 и равенства (3.13) получаем, что при $\varepsilon^{-1 / 2}<\rho<2 \varepsilon^{-1 / 2}$ (или, что то же самое, при $\varepsilon^{1 / 2}<r<2 \varepsilon^{1 / 2}$ )

$$
\begin{aligned}
V_{\varepsilon, N}(\xi)-K_{N}\left(\psi_{\varepsilon, N}^{\text {in }, \mathrm{ex}}(x, \tau(\varepsilon))\right) & =K_{N}\left(\psi_{\varepsilon}^{\text {in }, \mathrm{ex}}(x, \tau(\varepsilon))-\psi_{\varepsilon, N}^{\mathrm{in}, \mathrm{ex}}(x, \tau(\varepsilon))\right) \\
& =O\left(\varepsilon^{N} \ln ^{[N / 2]+1} \varepsilon \rho^{-5}\right)
\end{aligned}
$$

Причем это равенство можно дифференцировать.

Из (3.23) и (3.24) получаем, что

$$
\begin{aligned}
\left|V_{\varepsilon, N}\left(\frac{x}{\varepsilon}\right)-\psi_{\varepsilon, N}^{\text {in }}(x, \tau(\varepsilon))-\psi_{\varepsilon, N}^{\mathrm{ex}}(x, \tau(\varepsilon))\right| & =O\left(\varepsilon^{(N+1) / 2}\right), \\
\left|\frac{\partial}{\partial x_{i}}\left(V_{\varepsilon, N}\left(\frac{x}{\varepsilon}\right)-\psi_{\varepsilon, N}^{\text {in }}(x, \tau(\varepsilon))-\psi_{\varepsilon, N}^{\mathrm{ex}}(x, \tau(\varepsilon))\right)\right| & =O\left(\varepsilon^{N / 2}\right)
\end{aligned}
$$

при $\varepsilon^{1 / 2}<r<2 \varepsilon^{1 / 2}$. Из (3.25) следует, что и последнее условие леммы 3.4 вьполняется для $N_{1}=N / 2$. Следовательно, справедливо утверждение леммы 3.4 , причем $N_{2}=N / 2$ и имеет место сходимость (3.15).

Вначале рассуждением от противного докажем, что полюс имеет асимптотику (2.1), (2.2). Допустим, что существуют $\delta>0$ и $C>0$ такие, что $\left|\tau_{\varepsilon}-\tau(\varepsilon)\right|>C \varepsilon^{\delta}$. Тогда из (1.3), (1.4) и утверждения леммы 3.4 следует, что

$$
\left\|\psi_{\varepsilon, N}(\cdot, \tau(\varepsilon))\right\|_{1, S(R)}=O\left(\varepsilon^{N / 2-\delta}\right)
$$

для любого $R$, что в силу произвола в выборе $N$ противоречит (3.15). Следовательно, полюс $\tau_{\varepsilon}$ имеет асимптотику (2.1), (2.2).

Далее, из леммы 3.4, представления (1.3) и оценки (1.4) следует, что

$$
\psi_{\varepsilon, N}(x, k)=\alpha_{\varepsilon, N}(k) \Psi_{\varepsilon}(x)+\Psi_{\varepsilon, N}(x, k),
$$

где $\Psi_{\varepsilon, N}(x, k)$ - голоморфная функция из $W_{2,1 \text { ос }}^{1}\left(\mathbb{R}^{2}\right)$ (а следовательно, множитель $\alpha_{\varepsilon, N}(k)$ голоморфен), и

$$
\left\|\Psi_{\varepsilon, N}\left(\cdot, \tau_{\varepsilon}\right)\right\|_{1, S(R)}=O\left(\varepsilon^{N / 2}\right)
$$

для любого $R$. Из (3.15), (3.26) и (3.27) следует, что

$$
\psi_{\varepsilon, N}\left(x, \tau_{\varepsilon}\right)=\alpha_{\varepsilon, N}\left(\tau_{\varepsilon}\right) \Psi_{\varepsilon}(x)+O\left(\varepsilon^{N / 2}\right), \quad \alpha_{\varepsilon, N}\left(\tau_{\varepsilon}\right)=1+o(1) .
$$

Из (3.28), определения функции $\psi_{\varepsilon, N}$ и равенства (3.14) следует справедливость утверждения теоремы 2.1 для собственной функции вне $S\left(2 \varepsilon^{1 / 2}\right) \backslash S\left(\varepsilon^{1 / 2}\right)$. Но так как при $x \in S\left(2 \varepsilon^{1 / 2}\right) \backslash S\left(\varepsilon^{1 / 2}\right)$

$$
\begin{aligned}
\psi_{\varepsilon, N}\left(x, \tau_{\varepsilon}\right)= & V_{\varepsilon, N}\left(\frac{x}{\varepsilon}\right)+\left(1-\chi\left(r \varepsilon^{-1 / 2}\right)\right) \\
& \times\left(\psi_{\varepsilon, N}^{\text {in }}\left(x, \tau_{\varepsilon}\right)+\psi_{\varepsilon, N}^{\mathrm{ex}}\left(x, \tau_{\varepsilon}\right)-V_{\varepsilon, N}\left(\frac{x}{\varepsilon}\right)\right), \\
\psi_{\varepsilon, N}\left(x, \tau_{\varepsilon}\right)= & \psi_{\varepsilon, N}^{\text {in }}\left(x, \tau_{\varepsilon}\right)+\psi_{\varepsilon, N}^{\mathrm{ex}}\left(x, \tau_{\varepsilon}\right) \\
& -\chi\left(r \varepsilon^{-1 / 2}\right)\left(\psi_{\varepsilon, N}^{\text {in }}\left(x, \tau_{\varepsilon}\right)+\psi_{\varepsilon, N}^{\mathrm{ex}}\left(x, \tau_{\varepsilon}\right)-V_{\varepsilon, N}\left(\frac{x}{\varepsilon}\right)\right),
\end{aligned}
$$

то из (3.25) и (3.28) следует справедливость утверждения теоремы для собственной функции и при $x \in S\left(2 \varepsilon^{1 / 2}\right) \backslash S\left(\varepsilon^{1 / 2}\right)$. Теорема доказана. 
ЗАмЕчАниЕ 3.3. В силу произволав выборе $N$ легко видеть, что (3.7)-(3.9) являются полным асимптотическим разложением собственной функции $\psi_{\varepsilon}(x)$, равной $\alpha(\varepsilon) \Psi_{\varepsilon}(x)$ при некотором $\alpha(\varepsilon)$, стремящемся к единице при $\varepsilon \rightarrow 0$.

\section{§4. Построение асимптотик в случае симметричного отверстия}

В отличие от предыдушего параграфа ряды $\psi_{\varepsilon}^{\text {in,ex }}$ будем искать в виде

$$
\begin{aligned}
\psi_{\varepsilon}^{\mathrm{in}}(x, k)= & \left(k_{0}^{2}-k^{2}\right)\left(\sum_{j=0}^{\infty} \varepsilon^{2 j} R_{2 j+2}^{(0)}\left(D_{y}\right) G^{\mathrm{in}}(x, 0, k)\right. \\
& \left.+\sum_{j=1}^{\infty} \sum_{i=1}^{j} \varepsilon^{2 j} \ln ^{i} \varepsilon R_{2 j+1-2 i}^{(i)}\left(D_{y}\right) G^{\mathrm{in}}(x, 0, k)\right) \\
\psi_{\varepsilon}^{\mathrm{ex}}(x, k)= & \left(k^{2}-k_{0}^{2}\right)\left(\sum_{j=0}^{\infty} \varepsilon^{2 j} R_{2 j+2}^{(0)}\left(D_{y}\right) G^{\mathrm{ex}}(x, 0, k)\right. \\
& \left.+\sum_{j=1}^{\infty} \sum_{i=1}^{j} \varepsilon^{2 j} \ln ^{i} \varepsilon R_{2 j+1-2 i}^{(i)}\left(D_{y}\right) G^{\mathrm{ex}}(x, 0, k)\right),
\end{aligned}
$$

а асимптотики собственной функции - в виде (3.7), (3.8) и

$$
\psi_{\varepsilon}(x)=\sum_{j=2}^{\infty} \sum_{i=0}^{[(j-1) / 2]-1} \varepsilon^{j} \ln ^{i} \varepsilon v_{j, i}\left(\frac{x}{\varepsilon}\right) \text { при } x \in S\left(2 \varepsilon^{1 / 2}\right) .
$$

Краевые задачи для коэффициентов $v_{j, i}(\xi)$ получаются так же, как и в предыдущем параграфе, и имеют вид

$$
\begin{aligned}
& \Delta_{\xi} v_{j, i}=-\sum_{p=2}^{[j / 2]-2} \sum_{q=0}^{[p / 2]-2} \lambda_{2 p, q} v_{j-2 p-2, i-q}-\lambda_{0} v_{j-2, i} \quad \text { при } \xi \in \bar{\gamma}, \\
& v_{j, i}=0 \quad \text { при } \xi \in \gamma,
\end{aligned}
$$

где $\lambda_{p, q}$ - коэффициенты ряда $\lambda_{\varepsilon}=\tau_{\varepsilon}^{2}$ (но $\tau_{\varepsilon}-$ уже ряд вида (2.3)).

Обозначим через $Y^{\text {in,ex }}$ ряды вида

$$
\begin{aligned}
& Y^{\mathrm{in}}(\xi)=\rho^{4} \sin (2 \theta)+\sum_{j=1}^{\infty} c_{j} \rho^{-2 j+2} \sin (2 j \theta), \\
& Y^{\mathrm{ex}}(\xi)=\sum_{j=1}^{\infty} c_{j} \rho^{-2 j+2} \sin (2 j \theta) .
\end{aligned}
$$

С учетом последнего обозначения из леммы 3.1 и ее следствия вытекает следующий аналог леммы 3.5 .

ЛЕмма 4.1. Пусть асимптотика произвольной функиии $\tau(\varepsilon)$ имеет вид (2.3). Тогда для любого челого $N \geqslant 2$ справедливы равенства

$$
K_{N}\left(\psi_{\varepsilon}^{\mathrm{in}, \mathrm{ex}}\left(x, \tau_{\varepsilon}\right)\right)=\sum_{j=2}^{\infty} \sum_{i=0}^{[(j-1) / 2]-1} \varepsilon^{j} \ln ^{i} \varepsilon V_{j, i}^{\mathrm{in}, \operatorname{ex}}(\xi),
$$


где рядь $V_{j, q}^{\mathrm{in}, \mathrm{ex}}$ сопряжень при любъх фиксированных $j$ и

Рядыц $V_{j, 0}^{\mathrm{in}, \mathrm{ex}} \in \widetilde{\mathscr{B}}_{j}, V_{j, i}^{\mathrm{in}, \mathrm{ex}} \in \widetilde{\mathscr{B}}_{j-2 i-2}$ при $i>0$ являются формальньмми асимптотическими решениями (4.2) при $\rho \rightarrow \infty$, где в правой части уравнения функиии $v_{j, i}$ заменень на ряды $V_{j, i}^{\mathrm{in}, \mathrm{ex}}$. Четность рядов $V_{j, i}^{\mathrm{in}, \mathrm{ex}}$ по переменной $\xi_{1}$ совпадает с четностью $j+1$, и для них справедливы представления

$$
\begin{aligned}
& V_{2,0}^{\text {in }, \mathrm{ex}}(\xi)=\widetilde{V}_{2,0}^{\mathrm{in}, \mathrm{ex}}(\xi) \mp \tau_{4,0} \frac{2 k_{0}}{\pi}\left(a_{2,0}^{(2)} \rho^{-2} \sin (2 \theta)+\Pi_{0,0}\right), \\
& V_{2 j-1, i}^{\mathrm{in}, \mathrm{ex}}(\xi)=\widetilde{V}_{2 j-1, i}^{\mathrm{in}, \mathrm{ex}}(\xi) \mp \tau_{4,0} \frac{2 k_{0}}{\pi} \Pi_{2 j-3, i}, \\
& V_{2 j, i}^{\text {in }, \text { ex }}(\xi)=\widetilde{V}_{2 j, i}^{\text {in }, \text { ex }}(\xi) \mp \frac{2 k_{0}}{\pi}\left(\tau_{2 j+2, i}\left(a_{2,0}^{(2)} \rho^{-2} \sin (2 \theta)+\Pi_{0,0}\right)\right. \\
& \left.+\tau_{4,0} \Pi_{2 j-2, i}\right) \quad n p u \quad j \geqslant 2, \\
& \Pi_{2 m, i}=\sum_{q=2}^{\infty}(2 q-1) ! a_{2 m+2 q, i}^{(2 q)} \rho^{-2 q} \sin (2 q \theta) \text {, } \\
& \Pi_{2 m-1, i}=\sum_{q=1}^{\infty}(2 q-2) ! a_{2 m+2 q-2, i}^{(2 q-1)} \rho^{-2 q+1} \sin ((2 q-1) \theta) \\
& \widetilde{V}_{2,0}^{\text {in }}(\xi)=\frac{1}{2} a_{2,0}^{(2)} \psi_{2}^{2} \rho^{2} \sin (2 \theta), \quad \widetilde{V}_{3,0}^{\text {in }}(\xi)=\frac{1}{6} a_{2,0}^{(2)} \psi_{2} \psi_{3} \rho^{3} \sin (3 \theta) \\
& \widetilde{V}_{4,0}^{\text {in }}(\xi)=\frac{1}{4} a_{2,0}^{(2)} \psi_{2} \psi_{4} \rho^{4} \sin (4 \theta)+\frac{1}{2}\left(a_{4,0}^{(3)} \psi_{3}+a_{4,0}^{(4)}\left(6 \psi_{4}-\frac{1}{2} k_{0}^{2} \psi_{2}\right)\right) \psi_{2} \rho^{2} \sin (2 \theta) \\
& -\frac{1}{2} k_{0}^{2} a_{2,0}^{(2)} \psi_{2}^{2} Y^{\mathrm{in}}(\xi) \\
& \widetilde{V}_{2,0}^{\mathrm{ex}}(\xi)=\widetilde{V}_{3,0}^{\mathrm{ex}}(\xi) \equiv 0, \quad \widetilde{V}_{4,0}^{\mathrm{ex}}(\xi)=\frac{1}{2} k_{0}^{2} a_{2,0}^{(2)} \psi_{2}^{2} Y^{\mathrm{ex}}(\xi), \\
& \widetilde{V}_{6,1}^{\text {in }, \text { ex }}(\xi)= \pm \frac{1}{8} \pi^{-1} k_{0}^{5} \tau_{4,0} a_{2,0}^{(2)} \rho^{2} \sin (2 \theta) \text {. }
\end{aligned}
$$

Ряды $\widetilde{V}_{j, i}^{\mathrm{in}, \mathrm{ex}}$ не зависят от $\tau_{m+2, k}$ и $a_{m+q-2, k}^{(q)}$ при $m \geqslant j$.

Если к тому же $\operatorname{Im} a_{q+n, 0}^{(q)}=\operatorname{Im} \tau_{4,0}=0$ nрu $n \leqslant 2$, mo

$$
\begin{aligned}
& \operatorname{Im} \widetilde{V}_{5,0}^{\text {in }}(\xi)=0, \quad \operatorname{Im} \widetilde{V}_{6,0}^{\mathrm{in}}(\xi)=\frac{1}{2} \operatorname{Im} a_{6,0}^{(3)} \psi_{3} \psi_{2} \rho^{2} \sin (2 \theta), \\
& \operatorname{Im} \widetilde{V}_{5,0}^{\mathrm{ex}}(\xi)=2 k_{0} \tau_{4,0}\left(a_{2,0}^{(2)}\left(\left.\frac{\partial \operatorname{Im} G_{2}^{\mathrm{ex}}\left(x, 0, k_{0}\right)}{\partial x_{2}}\right|_{x=0}\right)\right. \\
& \left.+a_{2,0}^{(1)}\left(\left.\frac{\partial \operatorname{Im} G_{1}^{\mathrm{ex}}\left(x, 0, k_{0}\right)}{\partial x_{2}}\right|_{x=0}\right)\right) \rho \sin \theta, \\
& \operatorname{Im} \widetilde{V}_{6,0}^{\mathrm{ex}}(\xi)=k_{0} \tau_{4,0}\left(a_{2,0}^{(2)}\left(\left.\frac{\partial^{2} \operatorname{Im} G_{2}^{\operatorname{ex}}\left(x, 0, k_{0}\right)}{\partial x_{2} x_{1}}\right|_{x=0}\right)\right. \\
& \left.+a_{2,0}^{(1)}\left(\left.\frac{\partial^{2} \operatorname{Im} G_{1}^{\mathrm{ex}}\left(x, 0, k_{0}\right)}{\partial x_{2} x_{1}}\right|_{x=0}\right)\right) \rho^{2} \sin (2 \theta) .
\end{aligned}
$$

Прежде чем перейти к согласованию асимптотических разложений, докажем лемму 2.1 . 
ДокАЗАТЕЛЬСТво ЛЕммы 2.1. Обозначим $G_{q}(x)=G_{q}^{\mathrm{ex}}\left(x, 0, k_{0}\right)$. Согласно замечанию 3.1

$$
\operatorname{Im} G_{q}(x)=g_{q, 1} x_{1}+g_{q, 2} x_{1} x_{2}+O\left(r^{3}\right) \text { при } r \rightarrow 0
$$

Проинтегрировав по частям левые части равенств

$$
\operatorname{Im} \int_{B(R)} G_{q}\left(\Delta+k_{0}^{2}\right) \overline{G_{i}} d x=0, \quad \operatorname{Im} \int_{B(R)} G_{q}\left(\Delta+k_{0}^{2}\right) G_{i} d x=0
$$

и переходя к пределу при $R \rightarrow \infty$, получаем соотношения

$$
g_{q, j}+g_{j, q}=2 k_{0} \sigma_{q, j}, \quad g_{j, q}=g_{j, q}
$$

из которых следуют равенства

$$
g_{j, q}=k_{0} \sigma_{q, j}=k_{0} \sigma_{j, q}
$$

В силу этих равенств матрица $Q_{\Omega}$ с компонентами $g_{j, q}$ симметрична. Следовательно, сушествует ортогональная матрица $T$ с компонентами $t_{j, q}$ такая, что $D=T B_{\Omega} T^{\prime}$ - диагональная матрица с элементами $d_{q, q}\left(T^{\prime}\right.$ - транспонированная матрица). Обозначим

$$
g_{q}(x)=\sum_{i=1}^{2} t_{q, i} G_{i}(x)
$$

Проинтегрировав по частям левую часть равенства

$$
\int_{B(R)} g_{q}\left(\Delta+k_{0}^{2}\right) \bar{g}_{q} d x=0
$$

и перейдя к пределу при $R \rightarrow \infty$, получаем неравенства $d_{q, q}>0$, из которых следует справедливость леммы 2.1 .

Перейдем к согласованию асимптотических разложений (к доказательству аналога теоремы 3.1).

Теорема 4.1. Пусть $\omega_{-}=-\omega_{+}, N \geqslant 2-$ произвольное иелое число. Тогда существуют функиия $\tau(\varepsilon)$, имеющая асимптотику (2.3), и ряды $\psi_{\varepsilon}^{\text {in,ex } u(4.1)}$ такие, что коэффициенты $v_{j, 0} \in \mathscr{B}_{j}, v_{j, i} \in \mathscr{B}_{j-2 i-2}($ при $i>0)$ являются решениями краевых задач (4.2), при $\rho \rightarrow \infty$ справедливы равенства (3.13), коэффициенты $a_{2,0}^{(2)}$ и $v_{2,0}$ определяются соотношениями (3.14), коэффициенты $\tau_{4,0}, \tau_{6,0}, \tau_{8,1}, \tau_{8,0}$ удовлетворяют соотношениям (2.4) $и$

$$
a_{2,0}^{(1)}=\frac{1}{3} \psi_{3} \psi_{2}^{-1} c_{3,1} c_{2,2}^{-1}
$$


ДоКАЗАТЕЛЬСТво. Постоянную $a_{2,0}^{(2)}$ и функцию $v_{2,0}(\xi)$ выбираем из тех же соображений, что и при доказательстве теоремы 3.1. Учитывая следствие 2 леммы 3.6 и лемму 4.1 , при $\rho \rightarrow \infty$ имеем

$$
\begin{aligned}
& v_{2,0}(\xi)-V_{2,0}^{\mathrm{in}, \mathrm{ex}}(\xi)= \pm\left(\frac{1}{2} a_{2,0}^{(2)} \psi_{2}^{2} \sum_{q=1}^{\infty} c_{2,2 q} \rho^{-2 q} \sin (2 q \theta)\right. \\
& \left.\quad+2 k_{0} \tau_{4,0} \pi^{-1} \sum_{q=1}^{\infty}(2 q-1) ! a_{2 q, 0}^{(2 q)} \rho^{-2 q} \sin (2 q \theta)\right) \text { при } \pm \xi_{2} \geqslant 0 .
\end{aligned}
$$

Приравнивая в последнем равенстве коэффициенты при $\rho^{-2}$, получаем для $\tau_{4,0}$ значение (3.17), а затем с учетом значения $c_{2,2}$ - значение (2.4). Приравнивая коэффициенты при остальных степенях $\rho$, определяем вещественные постоянные $a_{2 q, 0}^{(2 q)}$ для $q \neq 1$ и, в частности, равенства $(4.4)$ и

$$
a_{4,0}^{(4)}=\frac{1}{6} \psi_{2}^{-1} c_{2,4} c_{2,2}^{-1}
$$

Положив $v_{3,0}=\frac{1}{6} \psi_{3} Y_{3}$, в силу леммы 4.1 и значения $(3.14)$ для $a_{2,0}^{(2)}$ имеем:

$$
\begin{aligned}
v_{3,0}(\xi)-\widetilde{V}_{3,0}^{\mathrm{in}, \mathrm{ex}}(\xi)= & \pm\left(\frac{1}{6} \psi_{3} \sum_{q=1}^{\infty} c_{3,2 q-1} \rho^{-2 q+1} \sin ((2 q-1) \theta)\right. \\
& \left.+2 k_{0} \tau_{4,0} \pi^{-1} \sum_{q=1}^{\infty}(2 q-2) ! a_{2 q, 0}^{(2 q-1)} \rho^{-2 q+1} \sin ((2 q-1) \theta)\right)
\end{aligned}
$$

Приравнивая правую часть последнего равенства к нулю, определяем вещественные $a_{2 q, 0}^{(2 q-1)}$ и, в частности, с учетом (3.17) получаем равенства (4.4) и

$$
a_{4,0}^{(3)}=\frac{1}{6} \psi_{3} \psi_{2}^{-2} c_{3,3} c_{2,2}^{-1}
$$

Дальнейшее доказательство проводится по индукции так же, как и в предыдущем параграфе, с использованием следствия 2 леммы 3.6 и леммы 4.1 (вместо леммы 3.5).

Покажем справедливость оставшихся равенств в (2.4). Краевая задача (4.2) для $v_{4,0}$ имеет вид

$$
\Delta v_{4,0}=-k_{0}^{2} v_{2,0} \quad \text { при } \xi \notin \bar{\gamma}, \quad v_{4,0}=0 \text { при } \xi \in \gamma .
$$

Из леммы 4.1 и проведенного согласования рядов $V_{2,0}^{\text {in, ex }}$ с асимптотиками на бесконечности функции $v_{2,0}$ следует, что ряды

$$
-\frac{1}{2} k_{0}^{2} a_{2,0}^{(2)} \psi_{2}^{2} Y^{\mathrm{in}}(\xi), \quad \frac{1}{2} k_{0}^{2} a_{2,0}^{(2)} \psi_{2}^{2} Y^{\mathrm{ex}}(\xi)
$$

являются асимптотическими решениями (4.7) при $\xi_{2}>0$ и $\xi_{2}<0$ соответственно. С другой стороны, так как $\Delta=4 \frac{\partial^{2}}{\partial \zeta \partial \bar{\zeta}}$, то функция

$$
Y(\xi)=\frac{1}{24} \operatorname{Im}\left(\bar{\zeta}\left(\zeta^{3}+\left(\zeta^{2}-\omega^{2}\right)^{3 / 2}\right)\right),
$$


где $\omega=\omega_{+}=-\omega_{-}$, во-первых, является решением краевой задачи (3.11) при $F=Y_{2}$ и принадлежит $\mathscr{B}_{4}$, а, во-вторых, ее асимптотика на бесконечности имеет структуру рядов $Y^{\text {in }}$ и $-Y^{\text {ех } п р и ~} \pm \xi_{2}>0$. Пусть $X_{1}$ и $X_{2}-$ два ряда из $\widetilde{\mathscr{B}}_{m}$ таких, что $\Delta X_{1}=\Delta X_{2}$ и их разность не содержит членов вида $\rho^{j} \sin (j \theta)$ при любом целом $j$. Легко видеть, что в этом случае $X_{1}(\xi)=X_{2}(\xi)$. А так как по определению функций $Y$ и $v_{2,0}$, функция

$$
-\frac{1}{2} k_{0}^{2} a_{2,0}^{(2)} \psi_{2}^{2} Y(\xi)
$$

является решением (4.7) (а следовательно, ее асимптотики являются асимптотическим решением (4.7)), то ее асимптотики совпадают с $Y^{\mathrm{in}}$ и $-Y^{\mathrm{ex}}$ при $\pm \xi_{2}>0$. С учетом этого замечания в силу лемм 4.1, 3.6, следствий леммы 3.6 и определения функций $v_{2,0}, Y_{4}$ и $Y$ функция

$$
\begin{aligned}
v_{4,0}(\xi)= & \frac{1}{4} a_{2,0}^{(2)} \psi_{2} \psi_{4} Y_{4}(\xi)+\frac{1}{2}\left(a_{4,0}^{(3)} \psi_{3}+a_{4,0}^{(4)}\left(6 \psi_{4}-\frac{1}{2} k_{0}^{2} \psi_{2}\right)\right) \psi_{2} Y_{2}(\xi) \\
& -\frac{1}{2} k_{0}^{2} a_{2,0}^{(2)} \psi_{2}^{2} Y(\xi)
\end{aligned}
$$

во-первых, является решением краевой задачи (4.7), а во-вторых, при $\rho \rightarrow \infty$ и $\pm \xi_{2} \geqslant 0$ справедливо равенство

$$
\begin{aligned}
0= & v_{4,0}(\xi)-V_{4,0}^{\mathrm{in}, \mathrm{ex}}(\xi)= \pm\left(\frac{1}{4} a_{2,0}^{(2)} \psi_{2} \psi_{4} c_{4,2}+\frac{1}{2}\left(a_{4,0}^{(3)} \psi_{3}\right.\right. \\
& \left.\left.+a_{4,0}^{(4)}\left(6 \psi_{4}-\frac{1}{2} k_{0}^{2} \psi_{2}\right)\right) \psi_{2} c_{2,2}+\frac{2 k_{0}}{\pi} \tau_{6,0} a_{2,0}^{(2)}\right) \rho^{-2} \sin (2 \theta)+O\left(\rho^{-3}\right) .
\end{aligned}
$$

Из равенства нулю коэффициентов при $\rho^{-2}$ и соотношений $(3.14),(4.5),(4.6)$ следует, что

$$
\tau_{6,0}=-\frac{\pi}{48 k_{0}}\left(6 \psi_{4} \psi_{2} c_{4,2}+2 \psi_{3}^{2} c_{3,3}+12 c_{2,4} \psi_{4} \psi_{2}-k_{0}^{2} \psi_{2}^{2} c_{2,4}\right)
$$

Подставляя в последнюю формулу значения постоянных $c_{3,3}, c_{4,2}$ и $c_{2,4}$, получаем (2.4) для $\tau_{6,0}$. Легко видеть, что коэффициенты $a_{q+2,0}^{(q)}$ вешественны.

Краевая задача (4.2) для $v_{6,1}$ имеет вид

$$
\Delta v_{6,1}=0 \text { при } \xi \notin \bar{\gamma}, \quad v_{6,1}=0 \text { при } \xi \in \gamma .
$$

Функция

$$
v_{6,1}(\xi)=\frac{1}{8 \pi} k_{0}^{5} \tau_{4,0} a_{2,0}^{(2)}\left(Y_{2}(\xi)+Y_{2}\left(\xi_{*}\right)\right)
$$

является решением этой задачи. В силу леммы 4.1 при $\rho \rightarrow \infty$ и $\pm \xi_{2} \geqslant 0$ справедливы равенства

$$
v_{6,1}(\xi)-V_{6,1}^{\mathrm{in}, \mathrm{ex}}(\xi)= \pm \frac{1}{\pi} k_{0} a_{2,0}^{(2)}\left(\frac{1}{4} k_{0}^{4} \tau_{4,0} c_{2,2}+2 \tau_{8,1}\right) \rho^{-2} \sin (2 \theta)+O\left(\rho^{-4}\right)
$$


Следовательно,

$$
\tau_{8,1}=-\frac{1}{8} k_{0}^{4} \tau_{4,0} c_{2,2}
$$

Подставляя в последнее выражение значения $\tau_{4,0}$ и $c_{2,2}$, получаем равенство (2.4) для $\tau_{8,1}$.

Так как по построению $\operatorname{Im} v_{2,0}=\operatorname{Im} v_{3,0}=\operatorname{Im} v_{4,0}=0$, то функции $\operatorname{Im} v_{5,0}$ и $\operatorname{Im} v_{6,0}$ являются решениями следуюших краевых задач

$$
\Delta \operatorname{Im} v_{j, 0}=0 \text { при } \xi \notin \bar{\gamma}, \quad \operatorname{Im} v_{j, 0}=0 \text { при } \xi \in \gamma .
$$

Так как по построению $\operatorname{Im} a_{q+n, 0}^{(q)}=\operatorname{Im} \tau_{4,0}=0$ при $n \leqslant 2$, то значение $\operatorname{Im} \tau_{8,0}$ определяем по следующей цепочке (см. лемму 4.1 и (4.3)):

$$
\begin{aligned}
& \operatorname{Im} \tilde{V}_{5,0}^{\text {in }}(\xi)= \\
& \operatorname{Im} \tilde{V}_{5,0}^{\text {ex }}(\xi)=2 k_{0} \tau_{4,0}\left(a_{2,0}^{(2)}\left(\left.\frac{\partial \operatorname{Im} G_{2}^{\mathrm{ex}}\left(x, 0, k_{0}\right)}{\partial x_{2}}\right|_{x=0}\right)\right. \\
& \quad \quad+a_{2,0}^{(1)}\left(\left.\frac{\partial \operatorname{Im} G_{1}^{\mathrm{ex}}\left(x, 0, k_{0}\right)}{\partial x_{2}}\right|_{x=0}\right) \rho \sin \theta \\
& \Rightarrow \operatorname{Im} v_{5,0}(\xi)=-2 k_{0} \tau_{4,0}\left(a_{2,0}^{(2)} \sigma_{2,1}+a_{2,0}^{(1)} \sigma_{1,1}\right) Y_{1}\left(\xi_{*}\right) \\
& \Rightarrow-2 k_{0}^{2} \tau_{4,0}\left(a_{2,0}^{(2)} \sigma_{2,1}+a_{2,0}^{(1)} \sigma_{1,1}\right) c_{1,3}=-4 k_{0} \tau_{4,0} \pi^{-1} \operatorname{Im} a_{6,0}^{(3)} \\
& \Rightarrow \operatorname{Im} a_{6,0}^{(3)}=\frac{1}{2} k_{0} \pi\left(a_{2,0}^{(2)} \sigma_{2,1}+a_{2,0}^{(1)} \sigma_{1,1}\right) c_{1,3}
\end{aligned}
$$

А так как согласно лемме 4.1 и равенству (4.3)

$$
\begin{aligned}
& \operatorname{Im} \widetilde{V}_{6,0}^{\text {in }}(\xi)=\frac{1}{2} \operatorname{Im} a_{6,0}^{(3)} \psi_{3} \psi_{2} \rho^{2} \sin (2 \theta) \\
& \operatorname{Im} \widetilde{V}_{6,0}^{\mathrm{ex}}(\xi)=k_{0}^{2} \tau_{4,0}\left(a_{2,0}^{(2)} \sigma_{2,2}+a_{2,0}^{(1)} \sigma_{1,2}\right) \rho^{2} \sin (2 \theta)
\end{aligned}
$$

то, учитывая значение (3.17) для $\tau_{4,0}$ и утверждение леммы 4.1 , получаем, что

$$
\begin{aligned}
\operatorname{Im} v_{6,0}(\xi)= & \frac{1}{4} k_{0} \pi\left(a_{2,0}^{(2)} \sigma_{2,1}+a_{2,0}^{(1)} \sigma_{1,1}\right) c_{1,3} \psi_{3} \psi_{2} Y_{2}(\xi) \\
& +\frac{1}{4} k_{0} \pi\left(a_{2,0}^{(2)} \sigma_{2,2}+a_{2,0}^{(1)} \sigma_{1,2}\right) \psi_{2}^{2} c_{2,2} Y\left(\xi_{*}\right) \\
\Rightarrow & -\frac{2 k_{0}}{\pi} a_{2,0}^{(2)} \operatorname{Im} \tau_{8,0}=\frac{1}{4} k_{0} \pi\left(\left(a_{2,0}^{(2)} \sigma_{2,1}+a_{2,0}^{(1)} \sigma_{1,1}\right) c_{1,3} \psi_{3} \psi_{2}\right. \\
& \left.+\left(a_{2,0}^{(2)} \sigma_{2,2}+a_{2,0}^{(1)} \sigma_{1,2}\right) \psi_{2}^{2} c_{2,2}\right) c_{2,2} \\
\Rightarrow & \operatorname{Im} \tau_{8,0}=-\frac{1}{8} \pi^{2} c_{2,2}\left(a_{2,0}^{(2)}\right)^{-1}\left(\left(a_{2,0}^{(2)} \sigma_{2,2}+a_{2,0}^{(1)} \sigma_{1,2}\right) \psi_{2}^{2} c_{2,2}\right. \\
& \left.+\left(a_{2,0}^{(2)} \sigma_{2,1}+a_{2,0}^{(1)} \sigma_{1,1}\right) c_{1,3} \psi_{3} \psi_{2}\right) .
\end{aligned}
$$

Подставляя в последнее равенство значения $c_{1,3}, c_{2,2}, a_{2,0}^{(2)}$ и $a_{2,0}^{(1)}$ (см. (3.14) и (4.4)), получаем формулу (2.4) для $\operatorname{Im} \tau_{8,0}$. Теорема доказана.

Обоснование построенных асимптотик, т.е. окончательное доказательство теоремњ 2.2, полностью аналогично обоснованию, приведенному в предыдущем параграфе при доказательстве теоремы 2.1. Главный член асимптотики собственной 
функции в $S\left(2 \varepsilon^{1 / 2}\right)$ определяется из (4.1) и значения (3.14) для $v_{2,0}$, а вид главного члена в $\Omega^{\text {ex }} \backslash S\left(\varepsilon^{1 / 2}\right)$ вытекает из структуры ряда $\psi_{\varepsilon}^{\text {ех }}$, равенств $(3.14)$ и (4.4) для его коэффициентов $a_{2,0}^{(2)}$ и $a_{2,0}^{(1)}$ и конкретных значений постоянных $c_{i, j}$ (с учетом того, что в рассматриваемом симметричном случае $\left.\varkappa_{\omega}=0\right)$.

\section{§5. Заключительные замечания}

Вырождение собственной функции в точке прорезания отверстия существенно влияет на асимптотики полюсов с малой мнимой частью и в случае классического акустического резонатора Гельмгольца (т.е. для задачи Неймана в $\mathbb{R}^{3}$ ). Построение же полных асимптотик для акустического резонатора сушественно проще, чем для рассматриваемого в настоящей работе его электромагнитного аналога, ввиду отсутствия в разложениях логарифмических членов [17].

Используя метод согласования асимптотических разложений, можно строить асимптотики полюсов, сходящихся к кратной собственной частоте предельной задачи. Случай, когда $k_{0}^{2}$ является двукратньм собственньм значением, рассмотрен в [18], где были приведены асимптотики двух полюсов, сходящихся к $k_{0}$ и имеющих разные порядки малости мнимых частей.

Отметим, что построение (и обоснование) асимптотик полюсов аналитического продолжения функции Грина (1.1), (1.2), сходящихся к полюсам аналитического продолжения функции Грина предельной внешней задачи, лежашим в нижней полуплоскости, не представляет каких-либо дополнительных трудностей (см., например, [19]). Правда, физический смысл таких полюсов неясен. Последнее, по-видимому, является причиной того, что аналитические продолжения решений в случае четномерных пространств обычно рассматриваются в комплексной плоскости с разрезом $\mathbb{C}^{\prime}$ (см., например, [20]). Хотя, вообше говоря, решение аналитически продолжается на бесконечнолистную поверхность $\widetilde{\mathbb{C}}$. Алгоритм построения асимптотик, приведенный в настояшей работе, сохраняется для любого листа $\widetilde{\mathbb{C}}$. Но так как значение функции Грина предельной внешней задачи при переходе с одного листа на другой изменяется, то, естественно, будут изменяться и значения коэффициентов в (2.1) и (2.3). Поэтому “проекции” полюсов, сходящихся к $k_{0}$ на разных листах $\widetilde{\mathbb{C}}$, не совпадут. Последнее, впрочем, неудивительно, так как известно, что такое несовпадение имеет место, например, для задачи рассеяния на круге [21].

В заключение заметим, что для резонаторов со стенками конечной толщины вопросы существования полюсов с малой мнимой частью, их резонансного характера, оценок и асимптотик рассматривались в [20], [22]-[30].

\section{Список литературы}

1. Wolfe P. An existence theorem for the reduced wave equation // Proc. Amer. Math. Soc. 1969. V. 21. P. 663-666.

2. Hayashi $Y$. The Dirichlet problem for the two-dimensional Helmholtz equation for an open boundary // J. Math. Anal. Appl. 1973. V. 44. P. 489-530.

3. Еремин Ю. А., Захаров E. В. О некоторых прямых и обратных задачах теории дифракции. Дополнение к кн.: Колтон Д., Кресс Р. Методы интегральных уравнений в теории рассеяния. М.: Мир, 1987.

4. Meixsner J. Die Kantenbedingung in der Theorie der Beugung electromagnetisher Wellen an volkommen lietenden ebenen Shirmer // Ann. Phys. 1949. V. 6. P. 1-9. 
5. Lord Rayleigh. The theory of Helmholtz resonator // Proc. Roy. Soc. London Ser. A. 1916. V. 92. P. 265-275.

6. Miles J. W. Scattering by a spherical cap // J. Acoust. Soc. Amer. 1971. V. 50. P. 892-903.

7. Sanchez-Hubert J., Sanchez-Palencia E. Vibration and coupling of continuous systems. Asymptotic methods. Berlin: Springer-Verlag, 1989.

8. Войтович Н. Н., Каценеленбаум Б. З., Сивов А. Н. Обобщенный метод собственных колебаний в теории дифракции. М.: Наука, 1977.

9. Шестопалов В. П. Сумматорные уравнения в современной теории дифракции. Киев: Наукова думка, 1983.

10. Готлиб В. Ю. Рассеяние на круглом резонаторе с узкой щелью как задача теории возмущений // Докл. АН СССР. 1986. Т. 287. С. 1109-1113.

11. Гадыльиин Р. Р. Поверхностные потенциалы и метод согласования асимптотических разложений в задаче о резонаторе Гельмгольца // Алгебра и анализ. 1992. Т. 4. № 2 . C. $88-115$.

12. Гадыльшин Р. Р. Существование и асимптотики полюсов с малой мнимой частью для резонатора Гельмгольца // УМН. 1997. Т. 52. № 2. С. 3-76.

13. Reichardt H. Ausstrahlungs Bedingungen fur die Wellengleichung // Abh. Math. Sem. Univ. Hamburg. 1960. V. 24. P. 41-53.

14. Ильин A. М. Согласование асимптотических разложений решений краевых задач. М.: Наука, 1989.

15. Колтон Д., Кресс Р. Методы интегральных уравнений в теории рассеяния. М.: Мир, 1987.

16. Эскин Г. И. Краевые задачи для эллиптических псевдодифференциальных уравнений. М.: Наука, 1973.

17. Гадыльшин P. Р. О влиянии выбора места отверстия и его формы на свойства акустического резонатора // ТМФ. 1992. Т. 93. № 1. С. 107-118.

18. Гадыльиин Р. Р. О расщеплении полюсов функции Грина для электромагнитного аналога резонатора Гельмголца // Радиотехника и электроника. Т. 36. С. 2045-2047.

19. Гадыльиин Р. Р. Об аналитическом продолжении функции Грина резонатора Гельмгольца // Асимптотические методы решения дифференциальных уравнений. Уфа, 1992. C. $18-26$.

20. Beale J. T. Scattering frequencies of resonators // Commun. Pure Appl. Math. 1973. V. 26. P. 549-563.

21. Shenk $N$., Thoe D. Resonant states and poles of scattering matrix for perturbations of $-\Delta$ // J. Math. Anal. Appl. 1972. V. 37. P. 467-491.

22. Арсеньев A. А. Об особенностях аналитического продолжения и резонансных свойствах решения задачи рассеяния для уравнения Гельмгольца // ЖВМиМФ. 1971. Т. 12. № 1. C. $112-138$.

23. Петрас С.В. О расщеплении серий резонансов на "нефизическом листе" // Записки научн. сем. ЛОМИ. 1975. Т. 51. С. 155-169.

24. Арсеньев $A$. A. О существовании резонансных полюсов и резонансов при рассеянии в случае краевых условий II и III рода // ЖВМиМФ. 1976. Т. 16. С. 718-724.

25. Санчес-Паленсиа E. Неоднородные среды и теория колебаний. М.: Мир, 1984.

26. Fernandez C. Resonances in scattering by a resonator // Indiana Univ. Math. J. 1985. V. 34. P. $115-125$.

27. Hislop P. D., Martinez A. Scattering resonances of a Helmholtz resonator // Indiana Univ. Math. J. 1991. V. 40. № 2. P. 767-788.

28. Hislop P. D. Singular perturbations of Dirichlet and Neumann domains and resonances for obstacle scattering // Astérisque. 1992. V. 210. P. 197-216.

29. Гадыльиин Р. Р. О полюсах акустического резонатора // Функц. анализ и его прилож. 1993. T. 27. № 5. C. 3-16.

30. Гадыльшин P. Р. Асимптотика решений сингулярно возмушенной задачи Дирихле // ЖВМиМФ. 1996. Т. 36. №1. С. 92-102. 